\title{
Calcineurin/NFAT Signaling Modulates Pulmonary Artery Smooth Muscle Cell Proliferation, Migration and Apoptosis in Monocrotaline-Induced Pulmonary Arterial Hypertension Rats
}

\author{
Rui-Lan He $\mathrm{H}^{\mathrm{a}, \mathrm{b}}$ Zhi-Juan Wu $\mathrm{W}^{\mathrm{a}, \mathrm{b}}$ Xiao-Ru Liu ${ }^{\mathrm{a}}$ Long-Xin Gui \\ Rui-Xing Wanga,b Mo-Jun Lin ${ }^{a, b}$ \\ aKey Laboratory of Fujian Province Universities on Ion Channel and Signal Transduction in \\ Cardiovascular Diseases, School of Basic Medical Sciences, Fujian Medical University, Fuzhou, \\ bDepartment of Physiology and Pathophysiology, School of Basic Medical Sciences, Fujian Medical \\ University, Fuzhou, China
}

\section{Key Words}

Pulmonary arterial hypertension $\bullet$ Calcineurin $\bullet$ Nuclear factor of activated T cells $\bullet$ Cyclosporine A - Monocrotaline

\begin{abstract}
Background/Aims: Pulmonary arterial hypertension (PAH) is a severe and debilitating disease characterized by remodeling of the pulmonary vessels, which is driven by excessive proliferation and migration and apoptosis resistance in pulmonary artery smooth muscle cells (PASMCs). The calcineurin $(\mathrm{CaN})$ /nuclear factor of activated T-cells (NFAT) signaling pathway is the most important downstream signaling pathway of store-operated $\mathrm{Ca}^{2+}$ entry (SOCE), which is increased in PAH. CaN/NFAT has been reported to contribute to abnormal proliferation in chronic hypoxia $(\mathrm{CH})$-induced PAH. However, the effect of CaN/NFAT signaling on PASMC proliferation, migration and apoptosis in monocrotaline (MCT)-induced PAH remains unclear. Methods: PAH rats were established by a single intraperitoneal injection of MCT for 21 days. PASMCs were isolated and cultured in normal and MCT-induced PAH Sprague-Dawley rat. PASMCs were treated with CsA targeting CaN and siRNA targeting NFATc2-4 gene respectively by liposome. We investigated the expression of calcineurin/NFAT signaling by immunofluorescence, QRT-PCR and Western blotting methods. Cell proliferation was monitored using MTS reagent or by assessing proliferating cell nuclear antigen (PCNA) expression. Cell apoptosis was evaluated with an Annexin V - FITC/propidium iodide (PI) apoptosis kit by flow cytometry. PASMC migration was assessed with a Transwell chamber. Results: MCT successfully induced PAH and pulmonary vascular remodeling in rats. CaN R.-L. He, Z.-J. Wu and X.-R. Liu contributed equally to this work.




\section{Cellular Physiology Cell Physiol Biochem 2018;49:172-189 \\ \begin{tabular}{l|l|l} 
DOI: 10.1159/000492852 & $\begin{array}{l}\text { O 2018 The Author(s). Published by S. Karger AG, Basel } \\
\text { www.karger.com/cpb }\end{array}$
\end{tabular}}

He et al.: Calcineurin/NFAT Signaling Modulates PASMC Proliferation, Migration and Apoptosis

phosphatase activity and nuclear translocation of NFATc2-4 were increased in PASMCs derived from MCT-treated rats. In addition, CaNBB/NFATc2-4 expression was amplified at the mRNA and protein levels. PASMC proliferation and migration were markedly inhibited in a dosedependent manner by cyclosporin A (CSA). Furthermore, siRNA targeting NFATc2 and NFATc4 attenuated the excessive proliferation and migration and apoptosis resistance in PASMCs derived from both CON and MCT-treated rats, while NFATc3 knockdown specifically affected MCT-PASMCs. Conclusion: Our results demonstrate that CaN/NFAT signaling is activated and involved in the modulation of PASMC proliferation, migration and apoptosis in MCT-induced PAH.

\section{Introduction}

Pulmonary arterial hypertension (PAH) is a severe and progressive disease characterized by increased pulmonary vascular resistance and arterial pressure, which lead to right ventricular hypertrophy and ultimately right heart failure [1-4]. A common trait related to PAH is sustained vascular remodeling of the distal pulmonary arteries $[4,5]$. The main pathological change is the thickening of the intimal and/or medial layer of muscular vessels, which results in obstruction of the vessel lumen and complex vascular lesions [46]. This change in vascular morphology subsequently causes increased pulmonary vascular resistance and decreased vascular compliance, which imposes a hemodynamic load on pulmonary artery pressure. Studies from various teams and our lab have indicated that abnormal cellular proliferation, migration and apoptosis of pulmonary artery smooth muscle cells (PASMCs) are closely associated with vascular remodeling in PAH [7-10]. Excessive proliferation and apoptosis resistance result in expansion of the PASMC population, while migration of PASMCs further causes arterial muscularization, vessel stenosis and increased vascular tension. These factors consequently increase in pulmonary artery pressure and promote the occurrence of $\mathrm{PAH}$.

The molecular and cellular mechanisms that drive maladaptive inward remodeling of pulmonary arteries have not been fully defined [5]. Current therapies for PAH are symptomatic treatment, which is mainly directed at vasodilatation. These therapies merely improve the symptomatic status or delay the progression of the disease but are not curative. Despite tremendous efforts by clinicians, the mortality rate of PAH remains unacceptably high $[11,12]$. Rectifying the proliferation, apoptosis and migration of PASMCs may be an effective approach to curb or reverse vascular remodeling in PAH and may thus provide a potential cure for this disease.

Intrinsic changes in $\mathrm{Ca}^{2+}$ homeostasis in PASMCs are known to be essential effecters of pulmonary vasoconstriction and vascular reconstruction in PAH [13-17]. An exciting new finding is that store-operated $\mathrm{Ca}^{2+}$ entry (SOCE), one of the main $\mathrm{Ca}^{2+}$ channels in the membrane of PASMCs regulating $\mathrm{Ca}^{2+}$ influx, modulates PASMC proliferation, apoptosis and migration in pulmonary hypertension $[18,19]$. The calcineurin $(\mathrm{CaN}) /$ nuclear factor of activated T- cells (NFAT) pathway is the most important downstream signaling pathway of SOCE and is involved in many physiological and pathological processes. CaN is a calcium- and calmodulin-dependent serine/threonine protein phosphatase. In response to increasing $\mathrm{Ca}^{2+}$ entry induced by SOCE, CaN docks with and dephosphorylates NFAT to permit its nuclear translocation and subsequent induction of transcriptional programs [20-23]. The NFAT family comprises five members, i.e., NFATc1, NFATc2, NFATc3, NFATc4, and NFAT5. NFATc1-4 are homologous, share similar regulatory pathways and are highly phosphorylated in the cytosol of quiescent cells [23-26]. The function of CaN/NFAT is very unclear and difficult to understand. Especially, each member of the NFAT family, the substrate of CaN, is very likely to play different even contrary roles under the same conditions [20, 25, 27-31]. Therefore, due to the uncertainty of the role of CaN/NFAT, highly controversial results are expected.

Although several studies have highlighted the importance of the CaN/NFAT pathway in $\mathrm{PAH}$, its exact effect on pulmonary artery remodeling is still puzzling [13, 32-36]. Current 


\section{Cellular Physiology Cell Physiol Biochem 2018;49:172-189 \\ \begin{tabular}{l|l} 
DOI: 10.1159/000492852 & $\begin{array}{l}\text { O 2018 The Author(s). Published by S. Karger AG, Basel } \\
\text { www.karger.com/cpb }\end{array}$
\end{tabular}}

He et al.: Calcineurin/NFAT Signaling Modulates PASMC Proliferation, Migration and Apoptosis

studies on chronic hypoxia (CH)-induced PAH have suggested that CaN/NFAT stimulates the constriction and proliferation of PASMCs, thereby aggravating vascular remodeling [35-37]. Our recent work using the monocrotaline (MCT)-induced PAH model demonstrated that the resting $\left[\mathrm{Ca}^{2+}\right]_{i}$ and SOCE level in PASMCs were markedly higher than those in controls [38]. These results strongly suggested the activation of the downstream CaN/NFAT pathway in MCT-induced PAH. However, to date, the role of CaN/NFAT in MCT-induced PAH is undefined.

$\mathrm{CH}$ and MCT are the two most widely used PAH models. Although the models cannot accurately reproduce human $\mathrm{PAH}$, they do provide insight into the signaling pathways that underlie the pathogenesis of PAH. As mimics of different subtypes of human PAH, these two models differ in their pathogenesis and pathological manifestations. The $\mathrm{CH}$ model in rodents is less severe and is usually considered to mimic hypoxia that occurs in pulmonary parenchymal disease, severe chronic obstructive pulmonary disease (COPD), and humans that reside at high altitude in humans. By contrast, the MCT model serves as an acute disease model characterized by acute/subacute damage of the peripheral vasculature of the lung and other organs (kidney, liver, and heart) [39, 40]. Due to the lack of data from MCT-induced $\mathrm{PAH}$, we cannot yet define the effect of the CaN/NFAT signaling pathway on PASMCs or the subsequent effects on vascular remodeling in PAH. Thus, further exploration is necessary.

This study was designed to investigate the effect of CaN/NFAT on PASMCs in the MCTinduced PAH rat model. Alterations in PASMC proliferation, migration and apoptosis in response to CaN/NFAT activation were measured by inhibiting overall CaN activity using its pharmacological inhibitor cyclosporin A (CsA). We subsequently identified the roles of each member of the NFAT family via NFAT small interfering RNA. The resulting new insights into the CaN/NFAT signaling pathway may reveal the underlying mechanism of vascular remodeling in PAH and lead to the identification of novel therapeutic targets.

\section{Materials and Methods}

\section{Animals and PAH model}

Experiments were performed on male Sprague-Dawley rats (170-180 g) supplied by the Animal Center of Fujian Medical University (Fuzhou, China). All procedures were approved by the Animal Care and Use Committee of Fujian Medical University. The animals were housed (5 rats per cage) with free access to food and water and a 12-hour light/dark cycle in a regulated room in which the ambient temperature was $24^{\circ} \mathrm{C}$ and the relative humidity was $45-50 \%$. PAH was induced by a single intraperitoneal injection of MCT (50 mg/kg, Sigma-Aldrich, St Louis, MO, USA) [9, 41]. The control (CON) group received saline injections. Twenty-one days after MCT injection, rats were anesthetized with an intraperitoneal injection of urethane $(1 \mathrm{~g} / \mathrm{kg})$. Right ventricular systolic pressure (RVSP) was measured by accessing the right ventricle through the jugular vein using a polyethylene catheter connected to a pressure transducer (YPJ01; Chengyi, Chengdu, China). Pressure signals were displayed continuously on an RM6240 polygraph (Chengyi, Chengdu, China). Following hemodynamic measurement, the rat was sacrificed with an overdose of urethane. The heart was then removed, and the weight of the left ventricle (LV), right ventricle (RV) and septum (S) and the right ventricular mass index (RVMI), which is the ratio of RV to $\mathrm{LV}$ plus $\mathrm{S}[\mathrm{RV} /(\mathrm{LV}+\mathrm{S})]$, were determined. Data were obtained from thirty-five rats in each group.

\section{Isolation and culture of PASMCs}

Rats were injected with heparin, anesthetized with urethane $(1 \mathrm{~g} / \mathrm{kg})$, and exsanguinated. The lungs were then removed and transferred to a petri dish filled with cold HEPES-buffered salt solution (HBSS) containing (in mM) $130 \mathrm{NaCl}, 5 \mathrm{KCl}, 1.2 \mathrm{MgCl}_{2}, 1.5 \mathrm{CaCl}_{2}, 10$ HEPES and 10 glucose, pH 7.2 (adjusted with $\mathrm{NaOH})$. Under a dissection microscope, the right and left branches of the main pulmonary artery were first isolated from the whole lung, and then, the third- and fourth-generation pulmonary arteries (PAs) ( $\sim 300$ to $800 \mu \mathrm{m})$ were isolated and cleared of connective tissue. The endothelium was removed by gently rubbing the luminal surface with a cotton swab. PASMCs were then enzymatically isolated and transiently cultured as previously described $[9,42]$. Briefly, arteries were allowed to recover for $30 \mathrm{~min}$ in cold HBSS, followed by $20 \mathrm{~min}$ in reduced-Ca ${ }^{2+}(20 \mu \mathrm{M}) \mathrm{HBSS}$ at room temperature. The tissue was digested at $37^{\circ} \mathrm{C}$ for $20 \mathrm{~min}$ in 20 


\section{Cellular Physiology Cell Physiol Biochem 2018;49:172-189

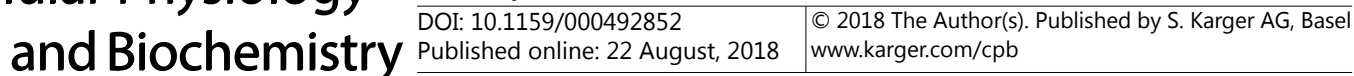

He et al.: Calcineurin/NFAT Signaling Modulates PASMC Proliferation, Migration and Apoptosis

$\mu \mathrm{M} \mathrm{Ca}^{2+} \mathrm{HBSS}$ containing collagenase (type $\left.\mathrm{I}, 1750 \mathrm{U} / \mathrm{ml}\right)$, papain $(9.5 \mathrm{U} / \mathrm{ml})$, bovine serum albumin $(2 \mathrm{mg} /$ $\mathrm{ml})$ and dithiothreitol $(1 \mathrm{mM})$ and then removed and washed with $\mathrm{Ca}^{2+}$-free HBSS to stop digestion. PASMCs were dispersed gently by agitation with a small-bore pipette in $\mathrm{Ca}^{2+}$-free HBSS at room temperature. The cell suspension was placed on 25-mm coverslips in Ham's F-12 medium (with L-glutamine) containing 10\% fetal calf serum (FBS, GIBCO, Carlsbad, CA, USA), $100 \mathrm{U} / \mathrm{ml}$ of streptomycin, and $0.1 \mathrm{mg} / \mathrm{ml}$ of penicillin (GIBCO, Carlsbad, CA, USA) and maintained at $37^{\circ} \mathrm{C}$ in $5 \% \mathrm{CO}_{2}$. The purity of the PASMCs in the primary cultures was confirmed via immunocytochemistry and immunofluorescence staining using a specific antibody against smooth muscle $\alpha$-actin. Cells from control rats were passaged 0 to 4 times, while primary PASMCs isolated from MCT-induced PAH rats were used in experiments. Cells at $80 \%$ confluence were used for all experiments.

\section{Immunohistochemistry}

Lung tissues were collected from rats in the experimental groups and fixed via the trachea with $4 \%$ paraformaldehyde for 24 hours. Then, lungs were embedded in paraffin blocks and cut into serial $6-\mu \mathrm{m}$ thick sections. Sections were stained with hematoxylin and eosin for light microscope observation. Images of the terminal arterioles were captured and processed with image analysis software (Image-Pro plus 6.0). The arterial diameters analyzed were classified as $<80 \mu \mathrm{m}, 80-150 \mu \mathrm{m}$ and $>150 \mu \mathrm{m}$, and the percent wall thickness was calculated using the ratio of wall thickness to vessel radius. Vessels from six rats in each group were measured.

\section{Quantitative real-time PCR}

Total RNA was extracted from PASMCs using TRIzol (Invitrogen, Carlsbad, CA, USA). cDNA was synthesized with the Transcriptor First Strand cDNA Synthesis Kit (Roche, Basel, Switzerland) using ramble oligo (dT) primers. To determine the expression levels of CaN and NFAT, qRT-PCR experiments were performed using the FastStart DNA Master SYBR Green I Kit and LightCycler 2.0 (Roche, Basel, Switzerland). Serially diluted solutions of $\beta$-actin cDNA-containing plasmids with a known copy number were used during each PCR experiment to perform a linear regression for analysis of the standard $\beta$-actin. All expression levels were normalized to the level of internal $\beta$-actin. The specific primers used for sequence detection are listed in Table 1 . The data were collected from independent cultures of rat cells $(n=5-18)$.

\section{Protein isolation and Western blotting}

The ProteinExt ${ }^{\mathrm{TM}}$ Mammalian Total Protein Extraction Kit and ProteinExt ${ }^{\mathrm{TM}}$ Mammalian Nuclear and Cytoplasmic Protein Extraction Kit (TransGen Biotech, Beijing, China) were used to isolate total, nuclear and cytoplasmic proteins from PASMCs. Protein concentrations were determined using a Pierce BCA protein assay kit (Thermo, Carlsbad, CA, USA). Proteins were separated on 8-12\% SDS-PAGE gels and transferred to a polyvinylidene fluoride membrane (Millipore, Billerica, MA, USA) using a liquid blotting system (Bio-Rad, Hercules, CA, USA). After blocking with 5\% skim milk in PBS for 2 hours, membranes were incubated with antibodies against proliferating cell nuclear antigen (PCNA) (Abcam, Cambridge, MA, USA, 1:100), CaNB $\beta$ (Proteintech, Wuhang, China, 1:200), NFATc3 (R\&D, Minneapolis, MN, USA, 1:200), NFATc4 (Santa Cruz, Dallas, TX, USA,1:200), $\beta$-actin (Cell Signaling, Danvers, MA, USA, 1:1000) and Lamin B1 (BD, San Jose, CA, USA, 1:1000) overnight at $4{ }^{\circ} \mathrm{C}$ and then with secondary antibodies (Cell Signaling, 1:5000) diluted using Secondary Antibody Dilution Buffer (Biyuntian, Shanghai, China) for 1 hour. Immunoreactive bands were detected using enhanced
Table 1. Oligonucleotide sequences of the primers used for RT-PCR

\begin{tabular}{|c|c|c|c|}
\hline Gene & $\mathrm{w}$ & Oligonucleotide Primers & Product (bp) \\
\hline$\beta$-actin & $\begin{array}{c}\text { Sense } \\
\text { Antisense }\end{array}$ & $\begin{array}{l}\text { 5'-CCCATCTATGAGGGTTACGC-3' } \\
\text { 5'-TTTAATGTCACGCACGATTTC-3' }\end{array}$ & 150 \\
\hline CaNA $\alpha$ & Sense & 5'- TACACGGTGGTTTGTCTCCA -3' & 175 \\
\hline CaNAB & $\begin{array}{c}\text { Antisense } \\
\text { Sense }\end{array}$ & $\begin{array}{l}\text { 5'- CAACCCCTGACTGTGTTGTG -3' } \\
\text { 5'-CAGCCCGGAAAGAAATCATA-3' }\end{array}$ & 127 \\
\hline CaNAY & $\begin{array}{c}\text { Antisense } \\
\text { Sense }\end{array}$ & $\begin{array}{l}\text { 5'-AACATCCCTGTGGGAGTCAG-3' } \\
\text { 5'-GAAGGAGCCACTTCAGTTCG-3' } \\
\text { 5'-GCCCTTGAGAATCAGCACAT-3' }\end{array}$ & 120 \\
\hline $\mathrm{CaNB} \alpha$ & $\begin{array}{c}\text { Sense } \\
\text { Antisense }\end{array}$ & $\begin{array}{l}\text { 5'-TCtTTGAGCGTGGAGGAGTT-3' } \\
\text { 5'-CGCCTTTGACACTGAACTGA-3' }\end{array}$ & 151 \\
\hline CaNB $\beta$ & $\begin{array}{c}\text { Sense } \\
\text { Antisense }\end{array}$ & $\begin{array}{l}\text { 5'-TCTTTGACACAGACGGCAAC-3' } \\
\text { 5'-GGTTGTTTCCCACCATCATC-3' }\end{array}$ & 189 \\
\hline NFATC1 & $\begin{array}{c}\text { Sense } \\
\text { Antisense }\end{array}$ & $\begin{array}{l}\text { 5'-ACCTGGCTTGGTAACACCAC-3' } \\
\text { 5'- GGGCTGTCTTTCGAGACTTG-3' }\end{array}$ & 124 \\
\hline NFATc2 & Sense & 5'-AAACAGGAGCAGAACCTGGA-3' & 177 \\
\hline NFATC3 & $\begin{array}{l}\text { Antisense } \\
\text { Sense }\end{array}$ & $\begin{array}{l}\text { 5'-ACGTGCAGTGTTCCAGTCAG-3' } \\
\text { 5'-GCAATCTCCTTGCCACTCTC-3' }\end{array}$ & 175 \\
\hline NFATC4 & $\begin{array}{c}\text { Antisense } \\
\text { Sense }\end{array}$ & 5'-CATGTAGTGGCCCCAGAAGT- $3^{\prime}$ & 236 \\
\hline NFAT5 & $\begin{array}{l}\text { Antisense } \\
\text { Sense } \\
\text { Antisense }\end{array}$ & $\begin{array}{c}\text { 5'-TCTTCGTCCCAGTCAGCTCT-3' } \\
\text { 5'-AGACTCTGACAACTATCCAAACGC-3 } \\
\text { 5'-TGCTGGACCAAGTCAACACTTC-3' }\end{array}$ & 361 \\
\hline
\end{tabular}




\section{Cellular Physiology Cell Physiol Biochem 2018;49:172-189 \\ \begin{tabular}{ll|l} 
DOI: 10.1159/000492852 & $\begin{array}{l}\text { O 2018 The Author(s). Published by S. Karger AG, Basel } \\
\text { www.karger.com/cpb }\end{array}$
\end{tabular}}

He et al.: Calcineurin/NFAT Signaling Modulates PASMC Proliferation, Migration and Apoptosis

chemiluminescence (ECL) reagent (Thermo, Carlsbad, CA, USA) to visualize the bands, and the optical density of each blot was normalized to that of $\beta$-actin or Lamin B1 and presented as relative optical density. The analyzed data were from three independent cultures of cells derived from nine rats.

\section{Immunofluorescence staining}

PASMCs were grown on coverslips, treated and fixed with $4 \%$ methanol. Cell membranes were ruptured with $0.1 \%$ Triton X-100. After blocking with immunology staining blocking buffer (Biyuntian, Shanghai, China), cells were incubated with antibodies against PCNA (Abcam, Cambridge, MA, USA, 1:200), NFATc2 (Abcam, Cambridge, MA, USA, 1:5 or 1:2), NFATc3 (Proteintech, Wuhan, China, 1:100 or 1:50), or NFATc4 (Santa Cruz, Dallas, TX, USA, 1:100 or 1:50). After overnight incubation, slides were washed and incubated with Alexa 488- or Alexa 555-conjugated secondary antibody (Biyuntian, Shanghai, China, 1:1000) for 1 hour and counterstained with DAPI (for nuclear staining). Fluorescence images were acquired using confocal laser scanning microscopy, and the corresponding protein expression levels were assessed.

\section{CaN phosphatase activity assay}

Primary PASMCs were successfully cultured and collected. Protein was precipitated with protein lysis buffer and centrifuged. Then, the supernatant was analyzed. CaN enzymatic activity was determined via a colorimetric method using a calcineurin cellular activity assay kit according to the instructions of the manufacturer (Jiancheng, Nanjing, China). CaN activity was assessed using the following formula: CaN viability $(\mathrm{U} / \mathrm{mg}$ prot $)=[$ (testing tube OD value - control tube OD value $) /$ standard tube OD value - standard blank tube OD value)] $\times$ concentration of standard tube $\times$ dilution multiple of reaction system $\times 3 /$ sample protein concentration. The experiments were repeated three times.

\section{Transfection with siRNA}

Small interfering RNA (siRNA) targeting NFATc2 (siNFATc2), NFATc3 (siNFATc3) and NFATc4 (siNFATc4) was synthesized by Genepharma (Shanghai, China) as follows:

NFATc2-siRNA: 5'-CCACUAGCUAUGAGAAGAUTT-3',

NFATc3-siRNA: 5'-GUCUCUCUCACACAUUUAUTT-3',

NFATc4-siRNA: 5'- GCAUCCGGAUCACCUCUAUTT-3'.

A mock treatment containing only transfection reagent was used as the control. PASMCs were transfected with siNFATc2-4 (final concentration of siRNA was $30 \mathrm{nM}$ ) using Lipofectamine RNAiMAX (Invitrogen, Carlsbad, CA, USA) according to the manufacturer's instructions. Following a 24-hour incubation period after transfection, cell proliferation, migration and apoptosis were assessed. The silencing efficiency was confirmed by qRT-PCR, which measured a greater than $70 \%$ decrease in mRNA, and by immunofluorescence experiments, which assessed protein levels.

Assessment of cell proliferation, migration and apoptosis

PASMC proliferation was quantified using CellTiter 96 AQueous MTS reagent (Promega, Madison, WI, USA) or by assessing PCNA (Abcam, Cambridge, MA, USA) expression. Apoptosis was evaluated with an Annexin V - FITC/propidium iodide (PI) apoptosis kit (BD, San Jose, CA, USA). PASMC migration was assessed with a Transwell chamber (Millipore, Billerica, MA, USA) with a pore diameter of $8 \mu \mathrm{m}$. The experiments were repeated three times.

\section{Solutions and drugs}

MCT powder was dissolved in $1 \mathrm{M} \mathrm{HCl}$, neutralized to $\mathrm{pH} 7.4$ with $1 \mathrm{M} \mathrm{NaOH}$, and diluted with saline. CsA was purchased from a hospital (50 mg/ml, Novartis Pharmaceuticals, Basel, Switzerland) and diluted with saline solution for injection or F12 solution containing 10\% FBS for immediate use. The CsA solution was stored at $4{ }^{\circ} \mathrm{C}$. The other stock solutions were stored at $-20^{\circ} \mathrm{C}$.

\section{Statistical analysis}

The data are presented as the mean \pm standard error of the mean (s.e.m.), and $\mathrm{n}$ indicates the number of animals or cell samples used. Curve fitting was performed using SigmaPlot 11.0 software (Systat Software Inc., Chicago, IL, USA). Statistical significance was assessed using unpaired or paired Student's t-tests and ANOVA wherever appropriate. Differences were considered statistically significant at $\mathrm{P}<0.05$ and $\mathrm{P}<0.01$. 


\section{Cellular Physiology

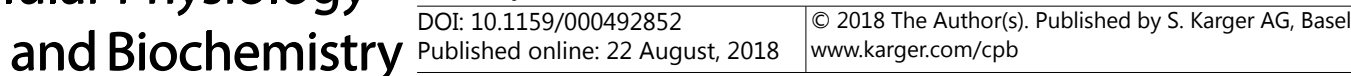

He et al.: Calcineurin/NFAT Signaling Modulates PASMC Proliferation, Migration and

Apoptosis

\section{Results}

MCT injection successfully induced PAH and pulmonary vascular remodeling in rats

As shown in Fig. 1, the RVSP, an index of pulmonary artery pressure (Ppa), was significantly higher in the MCT group than in the control (CON) group. Specifically, the RVSP values were $31.33 \pm 0.60 \mathrm{mmHg}$ and $83.05 \pm 1.59 \mathrm{mmHg}$ for the CON group and the MCT group, respectively $(\mathrm{n}=35$ each, $\mathrm{P}<0.01)$. The RVMI, also known as the RV/(LV $+\mathrm{S})$ mass ratios, increased from $0.26 \pm 0.003$ to $0.63 \pm 0.01$ in response to MCT treatment $(\mathrm{n}=$ 35 each, $\mathrm{P}<0.01$ ). In addition, the wall thickness of PAs of different diameters was much higher in MCT-treated rats than in CON rats. All of these findings indicated the successful establishment of the MCT-induced PAH rat model.

MCT treatment altered CaN/NFAT expression and activity in PASMCs

To study the role of CaN/NFAT in PAH, we first examined their mRNA and protein expression in PASMCs. CaN consists of five potential subunits, i.e., $\mathrm{CaNA} \alpha, \mathrm{CaNA} \beta, \mathrm{CaNA} \gamma, \mathrm{CaNB} \alpha$ and $\mathrm{CaNB} \beta$. Among them, CaNB $\beta$ expression was significantly increased at the mRNA and protein level in MCTPASMCs compared with CON-PASMCs (Fig. 2AC). CaN phosphatase activity was subsequently measured and demonstrated a dramatic 2.5 fold increase in MCT-PASMCs (Fig. 2D). The NFAT family also consists of five members, namely, NFATc1-4 and NFAT5. The mRNA levels of NFATc2-4 were significantly increased in MCT-PASMCs, while those of NFATc1 and NFAT5 were unaltered (Fig. 2E). Considering that nuclear translocation is required to activate NFAT-dependent transcription, we extracted and quantified the levels of cytoplasmic and nuclear NFAT protein in PASMCs. Compared with CON-PASMCs, NFATc3 and NFATc4 protein expression in MCT-PASMCs was markedly increased in the whole cell and in the cytoplasmic and nuclear fractions (Fig. $2 \mathrm{~F}$ and $2 \mathrm{G}$ ). In particular, protein expression of NFATc3 and NFATc4 in the nuclear fraction increased by $45.30 \%$ and $36.10 \%$ based on Western blotting results. However, NFATc2 protein expression in PASMCs was too low to be detected.

To clearly discriminate the localization of NFAT in the cytoplasm and nucleus, immunofluorescence examination was performed. As shown in Fig. 2HJ, immunofluorescence staining of NFATc2-4 was significantly increased in PASMCs derived from MCT-treated rats compared to those from control rats. More specifically, MCT treatment significantly stimulated nuclear translocation of NFATc2-4, which was indicated by strong NFATc2-4 staining in the nucleus and the ratio of nuclear to cytoplasmic NFATc2-4, which increased by $27.72 \%, 28.48 \%$ and $55.02 \%$, respectively (Fig. $2 \mathrm{~K}$ ).

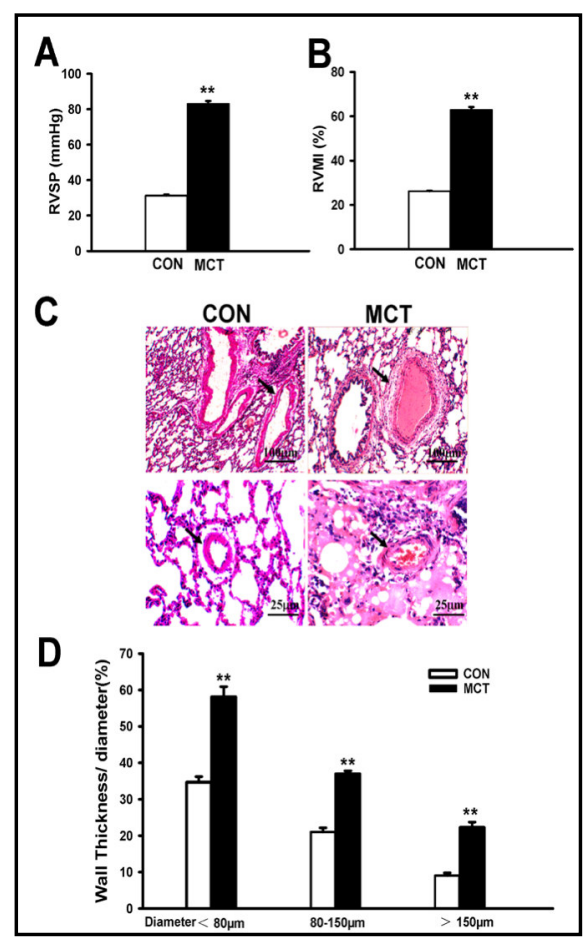

Fig. 1. Male Sprague-Dawley rats 170-180 $\mathrm{g}$ weight were injected intraperitoneally with saline solution or MCT $(50 \mathrm{mg} /$ $\mathrm{kg}$ ). After three weeks, pulmonary hypertension and pulmonary vascular remodeling were determined in MCTinjected rats. (A) Changes in Right ventricular systolic pressure (RVSP). (B) Changes in right ventricular mass index (RVMI). A and B data from $\mathrm{n}=35 \mathrm{CON}$ and MCT. (C) Representative images of lung sections of rats. Left panels from CON, right panels from MCT. Original magnification, above $\times 100$, below $\times 400$. (D) Changes in ratio of vessel wall thickness divided by vessel radius in pulmonary artery of CON and MCT-treated rats. $\mathrm{n}=6$ experiments each. Data are presented as mean \pm SEM. ** $\mathrm{P}<0.01$ vs $\mathrm{CON}$. 
Fig. 2. Calcineurin $(\mathrm{CaN})$ and NFAT isoforms were selectively upregulated and activated in PASMCs derived from MCT-treated rats. (A) Expression of CaN isforms mRNA in PASMCs with saline solution or MCT injection. MCT treatment only induced increased mRNA expression of CaNB $\beta$ normalized to $\beta$-actin mRNA level ( $\mathrm{n}=6-7 \mathrm{CON}$ and $\mathrm{n}=5-6 \mathrm{MCT}$ ). (B) Western blots showing CaNB $\beta$ and $\beta$-actin protein expression in the CON and MCT groups. (C) $\mathrm{CaNB} \beta$ protein was upregulated in MCT group normalized to $\beta$-actin protein level $(n=3$ per group). (D) CaN enzyme activity in MCT-PASMCs was determined by a CaN kit ( $\mathrm{n}=4$ per group). (E) Quantitative real-time RTPCR analysis of NFAT isoforms mRNA in CON- and MCT- PASMCs, normalized to $\beta$-actin mRNA level ( $\mathrm{n}=7-18$ CON and $\mathrm{n}=5-12 \mathrm{MCT}$ ). Western Blot showed that NFATc3 (F) and NFATc4 (G) protein from the whole cell (top left, normalized to $\beta$-actin protein level), cytoplasm (middle left, normalized to $\beta$-actin protein level) and nucleus (bottom left, normalized to Lamin B1 protein level) of PASMCs were all upregulated in MCT groups. Normalized amount of NFATc 3 and

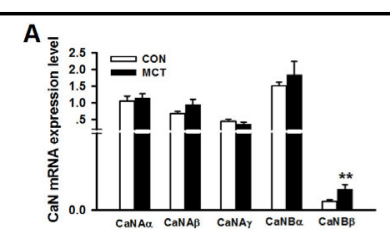

D

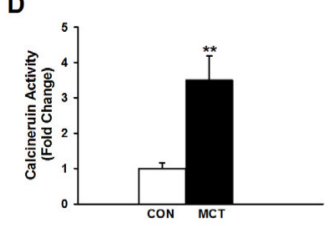

$F_{\text {Total }}$
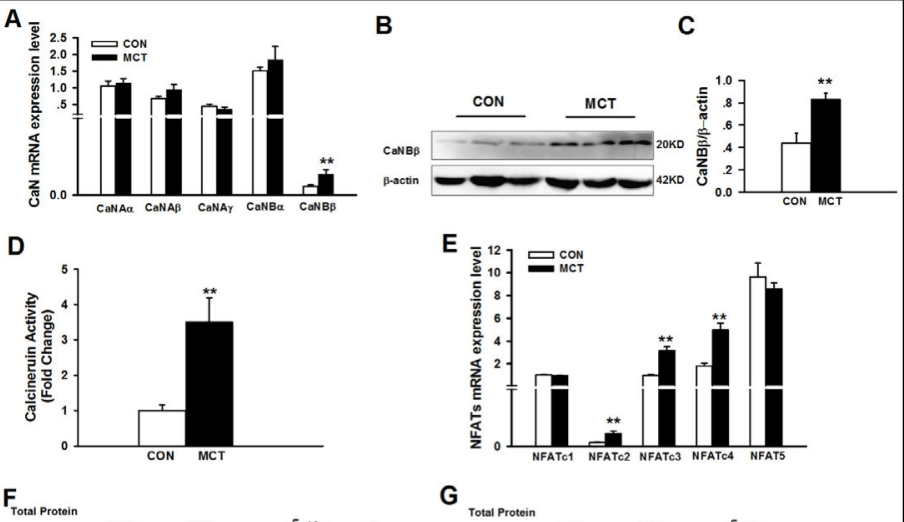

E
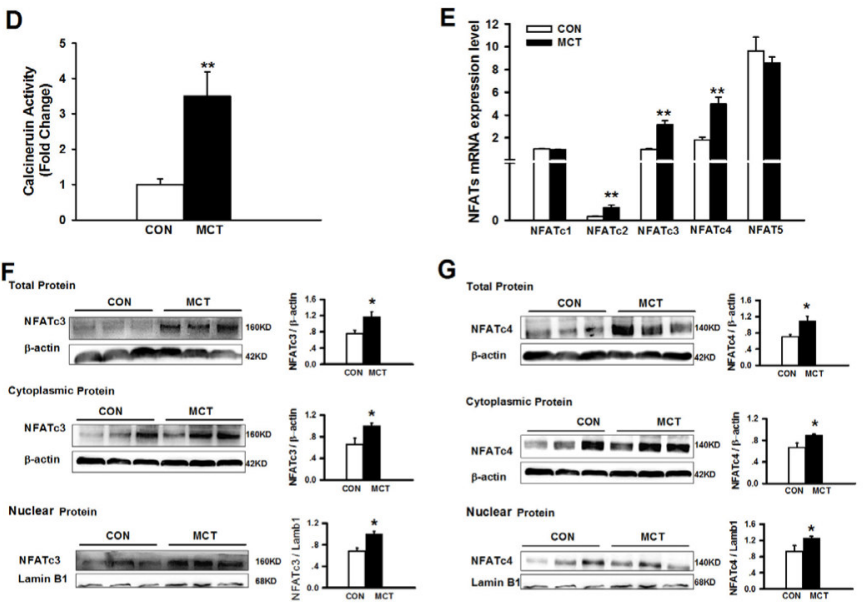

$\mathrm{H}$

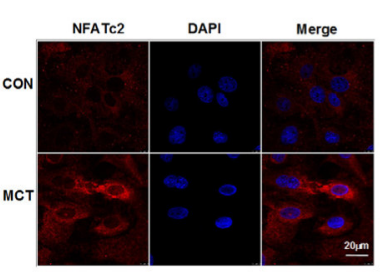

$\mathrm{J}$

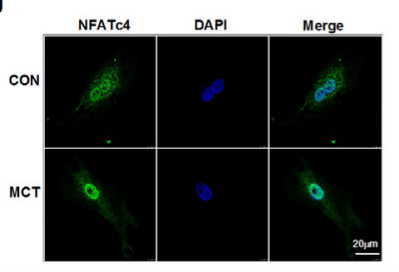

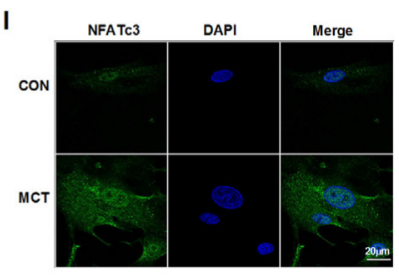

K

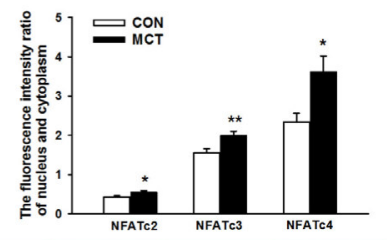

NFATc4 protein were calculated in

the right panel ( $\mathrm{n}=3$ per group). (H-J) Representative immunofluorescence images showing translocation of NFATc2 (red), NFATc3 (green), NFATc4 (green) into DAPI-stained nuclei (blue) in CON- and MCT-PASMCs. MCT treatment could improve the translocation of NFATc2, NFATc3 and NFATc4 in PASMCs cytoplasm to nucleus. (K) MCT-PASMCs had a high ratio of nucleus to cytoplasm of NFATc2-4 expression by randomly selecting $n=12$ cells from three independent cultures. Scale bars, $20 \mu \mathrm{m}$. Data are presented as mean \pm SEM. ${ }^{*} \mathrm{P}<0.05,{ }^{* *} \mathrm{P}<0.01$ vs $\mathrm{CON}$.

The above data, especially the increased CaN phosphatase activity and NFAT nuclear translocation, clearly verified the activation of CaN/NFAT signaling in PASMCs from MCTinduced PAH rats.

\section{MCT treatment enhanced PASMC proliferation and migration but inhibited apoptosis}

Compared with CON-PASMCs, MCT-PASMCs had significantly higher proliferation and migration rates and were more resistant to apoptosis. Enhanced MCT-PASMC proliferation was observed with an MTS assay. These results were confirmed by immunoblot analysis of PCNA, which showed a nearly two-fold increase in the MCT-treated group compared with the CON group (Fig. 3A). In addition to proliferation, there was a 4-fold increase in MCT-PASMC migration, as determined by Transwell chamber experiments (Fig. 3B). To further examine 
Fig. 3. Increased proliferation, migration and apoptotic resistance in MCT-PASMCs. (A) Proliferation of PASMCs was significantly potentiated in MCT-treated rats. PASMCs viability was assessed by MTS assay with OD $490 \mathrm{~nm}$ values (top, $\mathrm{n}=5$ each). Western blots showing PCNA and $\beta$-actin protein expression in CON and MCTPASMCs (middle). Increased PCNA protein expression in MCT-PASMCs normalized to $\beta$-actin protein (bottom, $\mathrm{n}=3$ each). (B) Migration to the bottom of the Transwell chambers in PASMCs of CON and MCT-treated rats. The number of

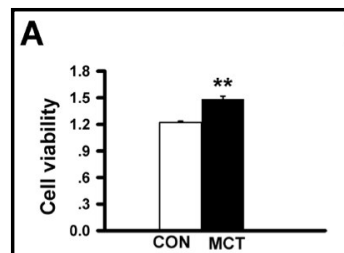

\section{B}
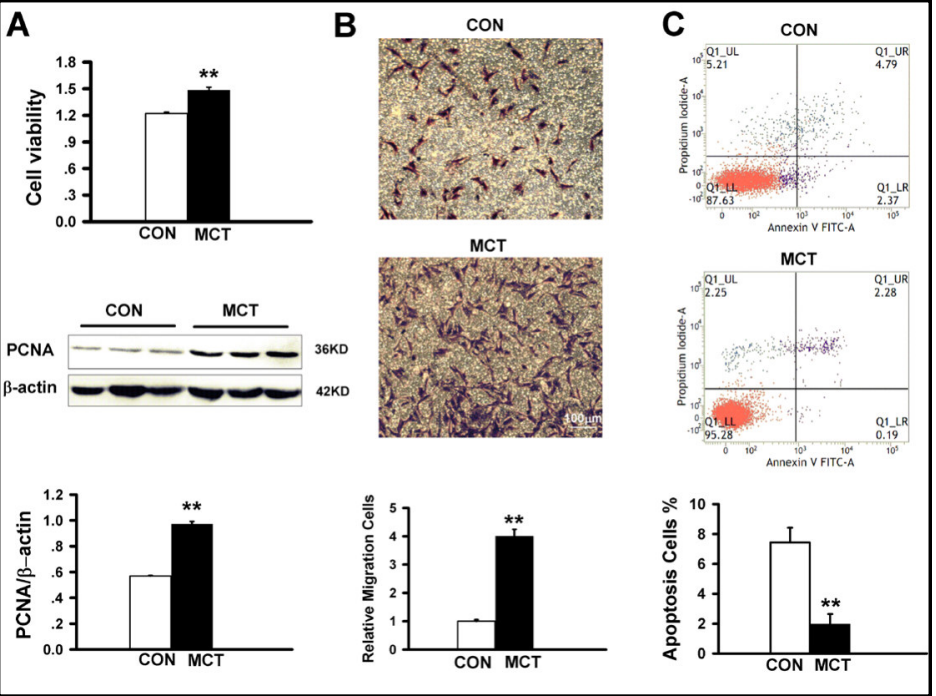

migrated cells was normalized to

CON-PASMCs. $n=5$ each. (C) Apoptosis was depressed in MCT- PASMCs measured by flow cytometer. $n=4$ each. Scale bars, $100 \mu \mathrm{m}$. Data are presented as mean \pm SEM. ${ }^{* * P}<0.01$ vs CON.

Fig. 4. Cyclosporine A (CsA) prevented NFAT nuclear translocation and activity in $\mathrm{CON}-$ and MCT-PASMCs. (A-C) and (EG) Confocal imaging of PASMCs representatively labeled with anti-NFATc2 (red), NFATc3 (green) and NFATc4 (green) in the CON and MCT groups with or without CsA treatment. DAPI-stained nuclei showed blue. (D) and (H) CsA treatment could decrease the translocation of NFATc2, NFATc3 and NFATc4 cytoplasm to nucleus in CON- and MCT PASMCs. (I) MCT-PASMCs with CsA treatment increased the inhibition of the nuclear translocation of NFATc2-4. Data are analyzed with randomly selecting $n=20$ cells from three independent cultures for each group. Scale bars, $100 \mu \mathrm{m}$. Data are presented as mean $\pm \mathrm{SEM} .{ }^{* *} \mathrm{P}$ $<0.01$ vs CON, \#\# $\mathrm{P}<0.01$ vs MCT. $\Delta \Delta \mathrm{P}<0.01$ vs CON treated with CsA.

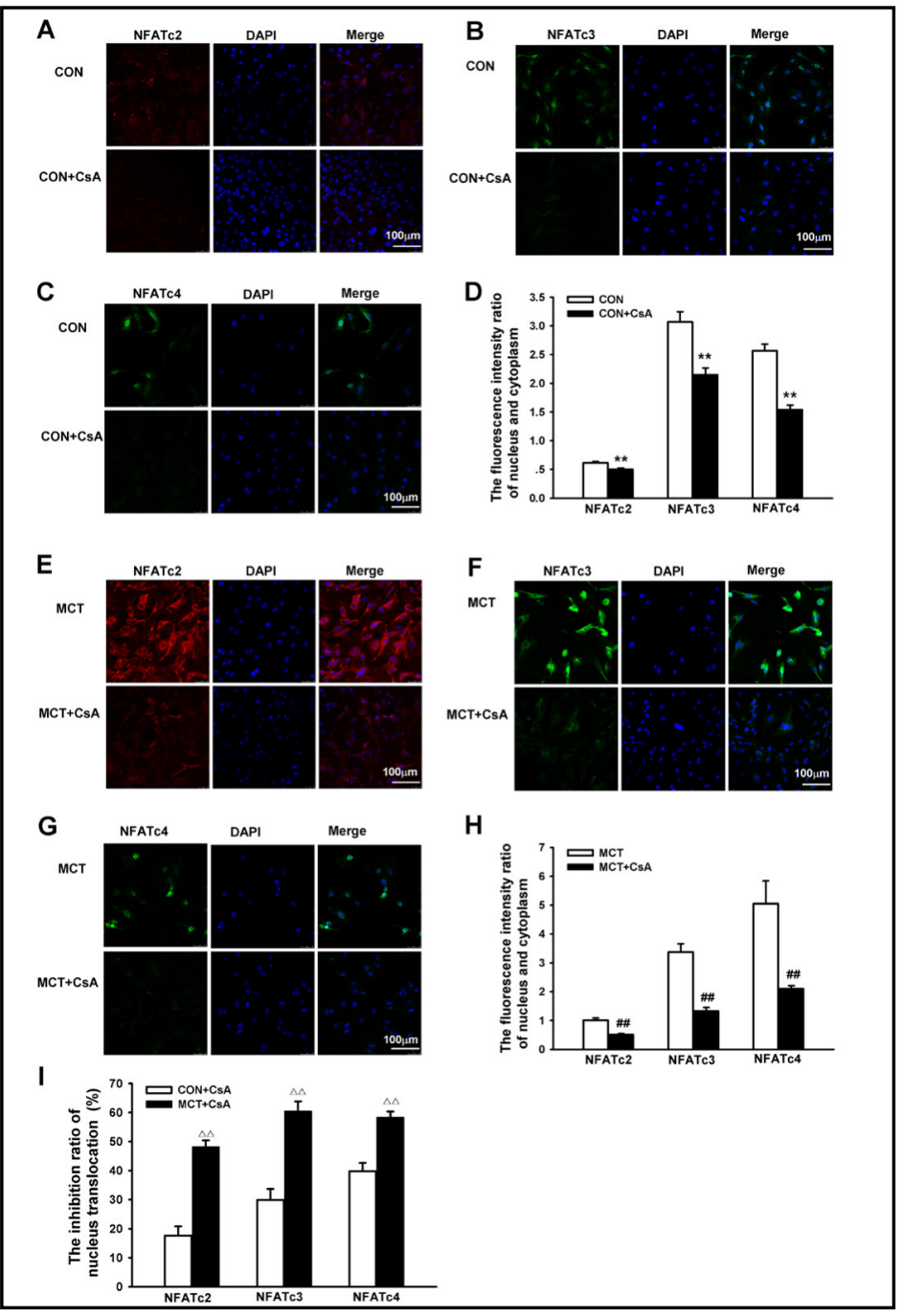


PASMC apoptosis in PAH, cells were detected by flow cytometry to evaluate Annexin V-FITC and PI labeling. Apoptosis in MCTPASMCs was approximately one-fourth that in CONPASMCs (Fig. 3C). These results suggested that MCT-induced PAH was associated with marked PASMC proliferation, migration and apoptosis suppression, all of which contribute to pulmonary artery remodeling and thus promote the development of PAH.

Effects of CsA on PASMC proliferation, migration and apoptosis in MCTinduced PAH

CsA, a pharmacological inhibitor of CaN, was used in this study to generally suppress the activation of the CaN/NFAT pathway. To confirm the inhibition of CaN/NFAT by CsA, we detected NFATc2-4 nuclear transport using immunofluorescence in the presence of $30 \mu \mathrm{M}$ CsA.

CsA was found to markedly inhibit NFATc2-4 nuclear translocation in PASMCs of the CON group (Fig. 4A-D) as well as in the those of the MCT group (Fig. 4E-H). Interestingly, MCT-PASMCs treated with CsA exhibited markedly impaired NFAT nuclear translocation (Fig. 4I), which was shown by the notably suppressed nuclear translocation rate in MCTPASMCs. As a result of CsA administration, nuclear translocation of NFATc2-4 respectively decreased by $48.13 \pm 2.32 \%, 60.40 \pm$ $3.42 \%$ and $58.33 \pm 1.99 \%$ in

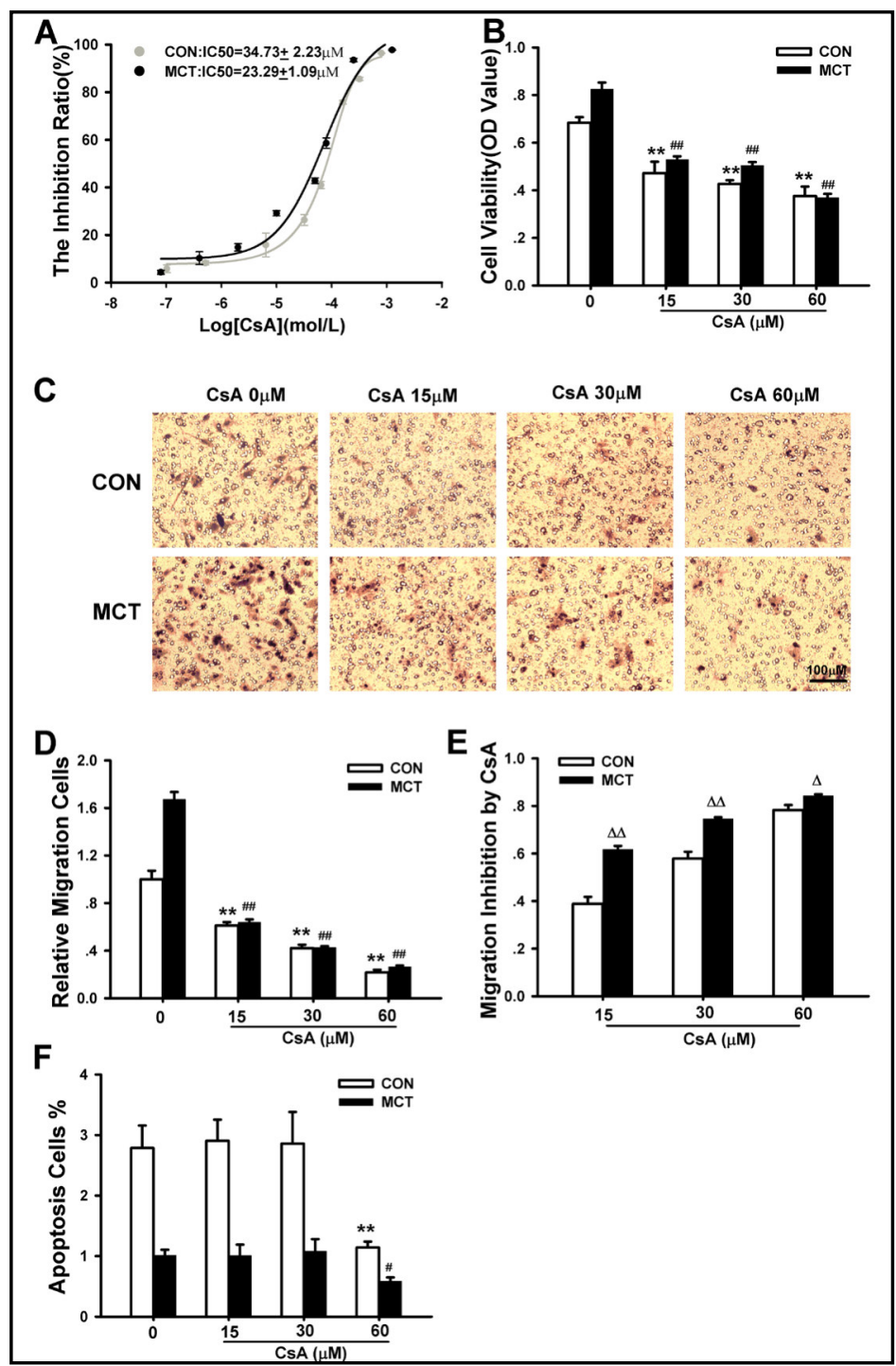

Fig. 5. CsA induced inhibition of proliferation and migration and apoptosis in PASMCs of CON and MCT-induced PAH rats. (A) Dosedependent effect of growth inhibition in PASMCs treated with CsA by MTS method in CON and MCT rats ( $\mathrm{n}=5$ each). (B) Proliferation of PASMCs was attenuated with three doses $(15,30$ and $60 \mu \mathrm{M})$ of CsA added to MCT-PASMCs ( $\mathrm{n}=4$ each). (C) Representative migration images demonstrating suppression of migration with CsA treatment in CON and MCT-PASMCs. (D) Compared with CON or MCT group without CsA intervention, three dosage CsA-pretreated groups all showed inhibition of migration in PASMCs. The number of migrated cells was normalized to CON-PASMCs. $\mathrm{n}=5$ each. (E) The migration inhibition rate of MCT-PASMCs with CsA was higher than that of CON-PASMCs with CsA. $n=5$ each. (F) Apoptosis of MCT-PASMCs was inhibited by $60 \mu \mathrm{M}$ CsA. Compared with CON or MCT group, $15 \mu \mathrm{M}$ and $30 \mu \mathrm{M}$ doses of CsA had no effect on the apoptosis in PASMCs, only high dose (60 $\mu \mathrm{M}) \mathrm{CsA}$ could attenuate the apoptosis of PASMCs $(\mathrm{n}=3-5)$. Scale bars, $100 \mu \mathrm{m}$. Data are presented as mean \pm SEM. ${ }^{* * P}<0.01$ vs CON without CsA treatment, \# $\mathrm{P}<0.05$, \#\# $\mathrm{P}<0.01$ vs MCT-PASMCs rats without CsA treatment. $\Delta \mathrm{P}<0.05, \Delta \Delta \mathrm{P}<0.01$ vs CON with CsA treatment. 


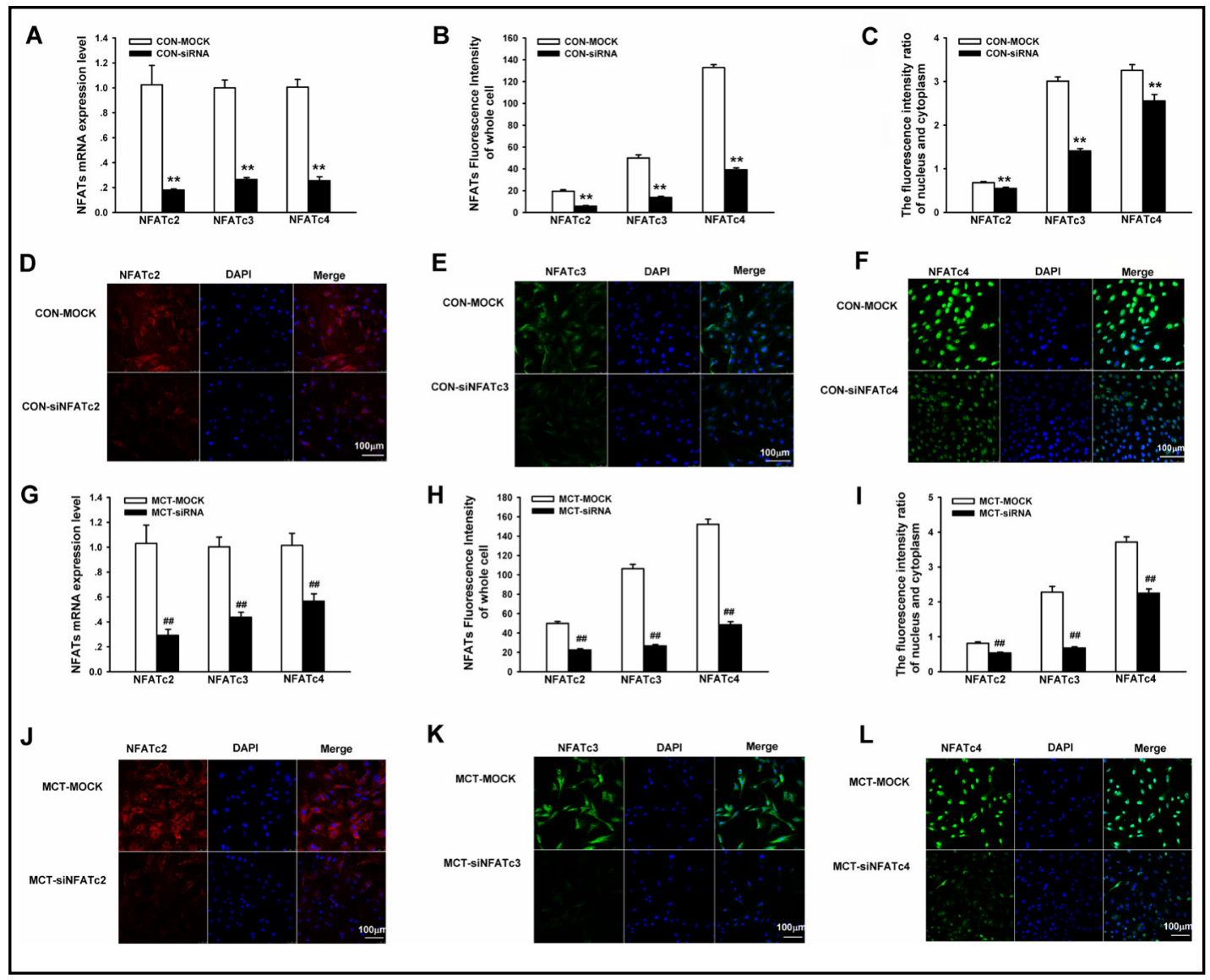

Fig. 6. NFAT knockdown reduced NFAT expression and nuclear translocation in CON- and MCT-PASMCs. After transfection with NFAT siRNA, PASMCs were cultured for 24 hours. (A) and (G) Real-time RT-PCR showing the reduction of NFATc2-4 by siRNA, respectively in the CON and MCT groups. Only adding lipofectamine transfection reagent as control (MOCK) and $\beta$-actin as loading control. $n=3$ each for NFATc2 gene expression, $n=4$ in CON-MOCK and $n=5$ in siNFATc3 for NFATc3 gene expression, $n=4$ in CONMOCK and $n=10$ in siNFATc4 for NFATc4 gene expression. (B) and (H) Decreasing expression of NFATc2-4 protein in the whole cell by immunofluorescence method after transfection with NFATs siRNA, respectively in the CON and MCT groups. Data are analyzed with randomly selecting $n=20$ cells from three independent cultures. (C) and (I) Silencing NFATc2-4 in PASMCs had a low ratio of nucleus to cytoplasm of NFATc2-4 expression in the CON and MCT groups by randomly selecting $\mathrm{n}=20$ cells from three independent cultures. (D-F) and (J-L) Representative immunofluorescence images of NFATc2 (red), NFATc3 (green), NFATc4 (green) into DAPI-stained nuclei (blue) in CON- and MCT-PASMCs with NFATs siRNA. Scale bars, $100 \mu \mathrm{m}$. Data are presented as mean \pm SEM. ${ }^{* *} \mathrm{P}<0.01$ vs CON-MOCK, \#\#P $<0.01$ vs MCT-MOCK.

MCT-PASMCs, whereas they decreased by only $17.60 \pm 3.19 \%, 29.86 \pm 3.81 \%$ and $39.78 \pm$ $2.88 \%$ in CON-PASMCs ( $\mathrm{n}=20$ each, $\mathrm{P}<0.01$ ).

We subsequently utilized CsA to roughly determine the effect of CaN/NFAT on PASMC proliferation, migration and apoptosis. PASMCs were treated with increasing concentrations of CsA $\left(10^{-7}\right.$ to $\left.10^{-3} \mathrm{M}\right)$ or vehicle solution for 24 hours. Proliferation of both CON- and MCTPASMCs was inhibited significantly in a concentration-dependent manner, with a half growth inhibition concentration $\left(\mathrm{IC}_{50}\right.$ ) of $34.73 \pm 2.23 \mu \mathrm{M}$ in CON-PASMCs that dropped to 23.29 $\pm 1.09 \mu \mathrm{M}$ in MCT-PASMCs based on MTS assays (Fig. 5A \& 5B). Similarly, CsA successfully suppressed the migration of PASMCs in both the CON and MCT groups. PASMC migration was more effectively inhibited by CsA in the MCT-induced PAH group than in the CON group (Fig. 5C and 5D). As shown in Fig. 5E, MCT-PASMC migration was decreased by $61.77 \%$, $74.52 \%$ and $84.21 \%$ in the presence of 15,30 , and $60 \mu \mathrm{M}$ CsA, respectively, which was much 
Fig. 7. Silencing NFATc2 by siRNA inhibited proliferation, migration and apoptosis resistance of PASMCs in the CON and MCT groups. After transfection with NFATc2 siRNA, PASMCs were cultured for 24 hours. Attenuated proliferation from PASMCs treated with lipofectamine transfection reagent (MOCK) or siRNA targeted to NFATc2 (siNFATc2) by MTS method (top left, $\mathrm{n}=5$ each) and immunofluorescence analysis of PCNA expression (top right, $\mathrm{n}=$ 40 each in CON and $n=37$ each in MCT) in CON (A) and MCT-treated (D) rats. PCNA staining was shown in green, and DAPI-stained nuclei was shown in blue (bottom). Suppressed migration to the bottom of the Transwell chambers in PASMCs transfected with siNFATc2 of CON (B) and PAH (E) rats. The number of migrated cells was normalized to CON-MOCK or
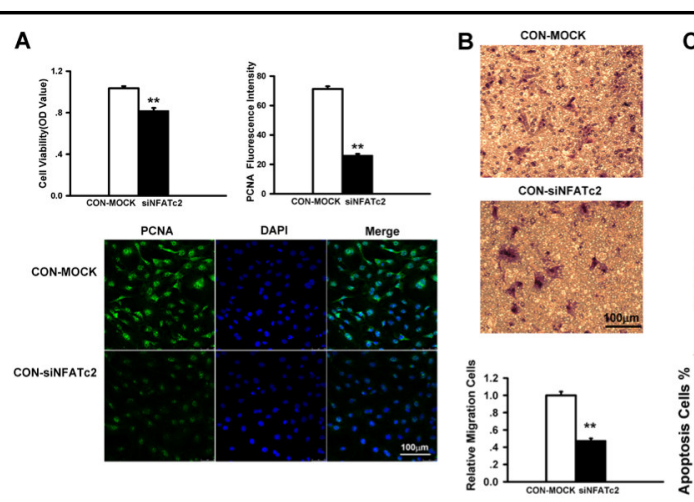

D
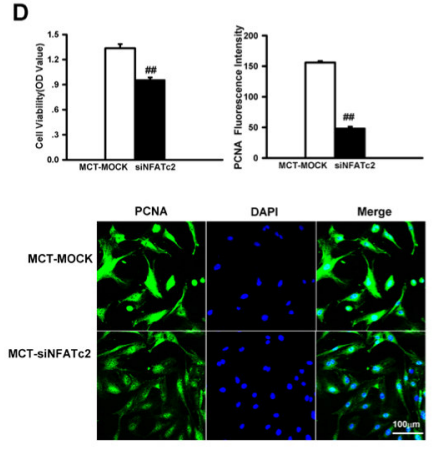
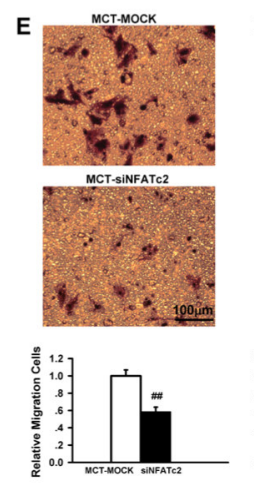
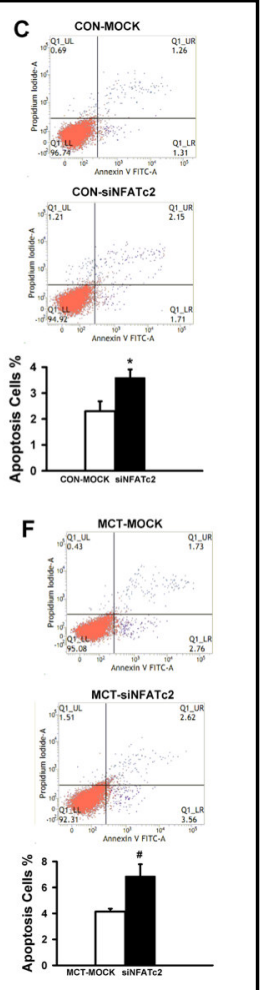

MCT-MOCK. $n=10$ each in CON and $n=5$ each in MCT. Apoptosis rate of PASMCs transfected with siNFATc2 from CON (C) and PAH (F) rats. $n=4$ each. Scale bars, $100 \mu$ m. Data are presented as mean \pm SEM. $* P<0.05$, $* * \mathrm{P}<0.01$ vs CON-MOCK and \#P $<0.05$, \#\#P $<0.01$ vs MCT-MOCK.

more severe than the decrease in migration observed in CON-PASMCs ( $\mathrm{n}=5, \mathrm{P}<0.05)$. To our surprise, CsA had almost no effect on PASMC apoptosis at the lowest and medium concentrations, i.e., 15 and $30 \mu \mathrm{M}$, whereas $60 \mu \mathrm{M}$ CsA inhibited apoptosis (Fig. 5F).

siRNA silencing of NFATC2-4 inhibited proliferation and migration but promoted apoptosis in PASMCS

The CsA experiments provided some information to roughly evaluate the role of CaN/ NFAT, we then further examined the contribution of the NFAT isoforms to the regulation of PASMC proliferation, migration and apoptosis by specifically and separately silencing NFATc2-4.

The efficacy of NFATc2-4 siRNA was verified by real-time PCR and immunofluorescence in CON- and MCT-PASMCs. Cells treated with lipofectamine transfection reagent only (MOCK) were used as controls. Specific siRNAs reduced NFATc2-4 mRNA levels (normalized to $\beta$-actin), as shown by real-time PCR analysis (Fig. 6A and 6G). A significant reduction in NFATc2-4 protein levels in whole cells was determined by immunofluorescence staining (Fig. 6B and 6H). Notably, targeted knockdown of NFATc2-4 sharply inhibited NFAT nuclear translocation (Fig. 6C and 6I). These inhibitory effects of siRNA are illustrated in fluorescence diagrams (Fig. 6D-F and 6J-L), which indicate the successful knockdown of NFAT in PASMCs.

NFATc2 siRNA non-selectively inhibited PASMC proliferation in both CON and MCTinduced PAH rats (Fig. 7A and 7D). Cell viability decreased by $20.87 \pm 2.63 \%$ and $28.26 \pm$ $2.06 \%$ in CON-PASMCs and MCT-PASMCs, respectively ( $\mathrm{P}=0.057$, CON $v s . \mathrm{MCT}, \mathrm{n}=5$ each). The results were confirmed by immunofluorescence staining of PCNA (Fig. 7A and 7D). Silencing NFATc2 also dramatically prevented PASMC migration in the CON $(52.42 \pm 2.63 \%$, $\mathrm{n}=10$ ) (Fig. 7B) and MCT (41.55 $\pm 5.37 \%, \mathrm{n}=5, \mathrm{P}=0.059)$ (Fig. 7E) groups. By contrast, NFATc2 knockdown enhanced PASMC apoptosis, as determined by flow cytometry analysis 
Fig. 8. Knockdown NFATc3 by siRNA attenuated proliferation, migration and apoptosis resistance of MCT-PASMCs. After transfection with NFATc3 siRNA, PASMCs were cultured for 24 hours. Knockdown NFATc3 had no effect on proliferation (A), migration (B) and apoptosis (C) of PASMCs in CON rats. The number of migrated cells was normalized to $\mathrm{CON}$ MOCK. Analysis of proliferation by MTS method ( $n=4$ each) and PCNA immunofluorescence ( $\mathrm{n}=$ 23 each). $n=10$ each for migration and $n=4$ each for apoptosis. (D) Suppressed proliferation from PAH PASMCs treated with lipofectamine transfection reagent (MOCK) or siRNA targeted to NFATc3 (siNFATc3) by MTS method (top left, $\mathrm{n}=5$ each) and immunofluorescence analysis of PCNA expression (top right, $\mathrm{n}=$ 33 each). (E) Inhibited migration

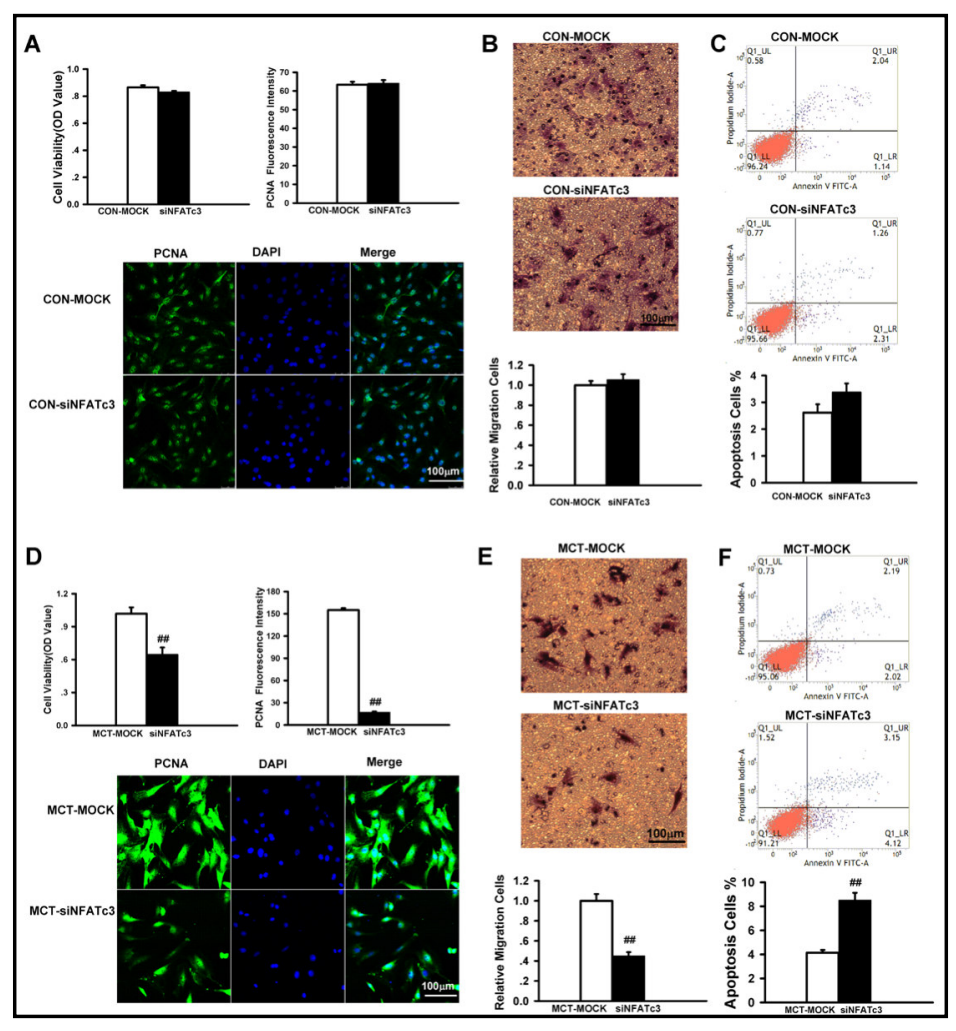
to the bottom of the Transwell chambers of PASMCs transfected with siNFATc3 in MCT-reduced PAH rats. The number of migrated cells was normalized to MCT-MOCK. $n=5$ each. (F) Increased apoptosis rate in PASMCs transfected with siNFATc3 from MCT-reduced PAH rats. $\mathrm{n}=4$ each. Scale bars, $100 \mu \mathrm{m}$. Data are presented as mean $\pm \mathrm{SEM}$. \#\#P $<0.01$ vs MCT-MOCK.

of the CON $(55.92 \pm 13.95 \%, n=4)$ (Fig. 7 C) and MCT groups $(65.57 \pm 22.45 \%, n=4, P=$ 0.734) (Fig. 7F).

In contrast with NFATc2, NFATc3 appeared to affect MCT-PASMCs only (Fig. 8A-C). According to MTS assays, NFATc3 knockdown effectively inhibited proliferation in the MCT-PASMCs by $36.43 \%$, and based on PCNA expression, NFATc3 knockdown inhibited proliferation in the MCT-PASMCs by $89.07 \%$ (Fig. 8D). Likewise, MCT-PASMC migration was decreased by $55.07 \%$ after NFATc3 silencing (Fig. 8E). Moreover, NFATc3 siRNA doubled the level of PASMC apoptosis in MCT-induced PAH (Fig. 8F). These data suggested that NFATc3 may contribute specifically to MCT-induced abnormalities in PASMC proliferation, migration and apoptosis.

Similar to NFATc2, NFATc4 affected PASMC proliferation, migration and apoptosis in both the CON and MCT groups. As a result of NFATc 4 knockdown, cell viability was decreased by $37.48 \pm 1.70 \%$ in the CON group (Fig. $9 \mathrm{~A}$ ) and $41.13 \pm 2.31 \%$ in the MCT-induced PAH group ( $\mathrm{n}=5$ each) based on MTS assays (Fig. 9D), and these results were also confirmed by PCNA fluorescence. NFATc 4 also inhibited CON-PASMC $(54.63 \pm 3.60 \%, n=10$, Fig. 9B) and MCT-PASMC migration ( $60.39 \pm 3.94 \%, n=5$, Fig. 9E). Furthermore, cellular apoptosis was increased by $81.98 \pm 16.44 \%$ in the CON group ( $n=4$, Fig. $9 \mathrm{C}$ ) and by $62.91 \pm 2.60 \%$ in the MCT-induced PAH group ( $\mathrm{n}=3$, Fig. 9F, $\mathrm{P}=0.375)$. 
Fig. 9. Silencing NFATc4 by siRNA reduced proliferation, migration and apoptosis resistance in CON and MCT-PASMCs. Inhibited proliferation from PASMCs treated with lipofectamine transfection reagent (MOCK) or siRNA targeted to NFATc4 (siNFATc4) by MTS method ( $\mathrm{n}=5$ each) and immunofluorescence analysis of PCNA expression ( $\mathrm{n}=51$ in CON, $\mathrm{n}=39$ in MCT) in CON (A) and MCT-PASMCs (D). Suppressed migration to the bottom of the Transwell chambers in PASMCs transfected with siNFATc4 of CON (B) and MCT-PASMCs (E), $n=5$ each. Enhanced apoptosis rate in PASMCs transfected with siNFATc4 from CON (C) and MCT-PASMCs (F). The number of migrated cells was normalized to that in the CONMOCK or MCT-MOCK groups. $\mathrm{n}=$ 4 each. Scale bars, $100 \mu \mathrm{m}$. Data are presented as mean \pm SEM. **P $<0.01$ vs CON-MOCK and \#\#P $<0.01$ vs MCT-MOCK.
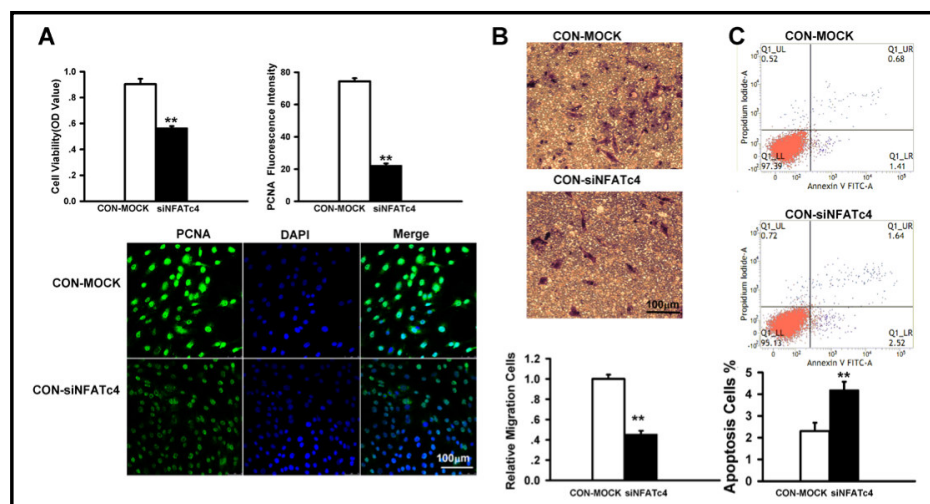

D

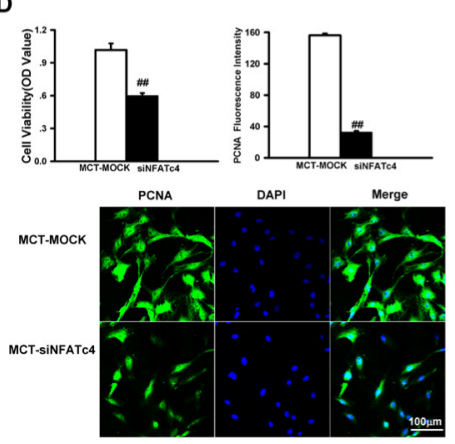

E
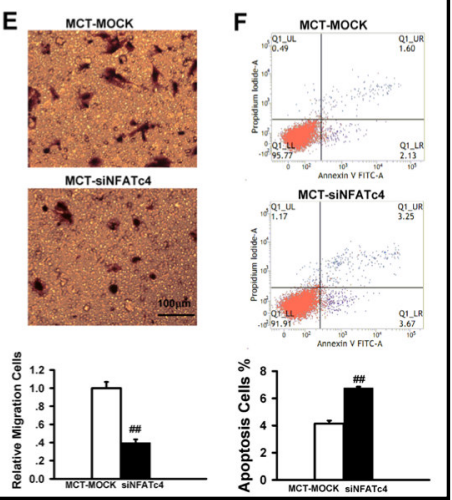

\section{Discussion}

In this study, we explored the effect of the CaN/NFAT signaling axis in the development of PAH using in vitro cultured PASMCs derived from MCT-induced PAH rats. We found that CaN/NFAT signaling was significantly activated 21 days after MCT injection, as evidenced by a 2.5 -fold increase in CaN activity and a more than 25-percent increase in NFATc2-4 nuclear translocation. Similarly, the expression of CaN/NFAT signaling was also significantly increased. CsA, a pharmacological inhibitor of $\mathrm{CaN}$, markedly reduced PASMC proliferation and migration in both the CON and MCT groups, while it slightly inhibited cellular apoptosis at a dose of $60 \mu \mathrm{M}$. We further identified the specific functions of NFATc2-4. Interestingly, siRNAs targeting NFATc2 and NFATc4 repressed PASMC proliferation and migration while enhancing apoptosis in both the CON and PAH groups. NFATc3 silencing had similar effects, but specifically on MCT-PASMCs. All the results suggested that CaN/NFAT signaling modulates the proliferation, migration and apoptosis of PASMCs and is thus associated with the development of PAH.

The pathogenesis of PAH is characterized by pulmonary vascular remodeling with stiffening of the elastic proximal pulmonary arteries and thickening of the intimal and/or medial layer of muscular arteries, resulting from aberrant PASMC proliferation, migration and apoptosis $[4,43,44]$. In this study, excessive proliferation and migration and apoptosis resistance were observed in the in vitro cultured PASMCs derived from MCT-treated rats. Compared with CON-PASMCs, MCT-PASMCs exhibited $21.43 \%$ and $70.40 \%$ increased cellular viability, as measured by MTS assay and PCNA expression analysis, respectively. There was also a 4-fold increase in migration and an approximately 25\% decrease in apoptosis of MCTPASMCs. These results indicated that the homeostasis of PASMC proliferation, migration and apoptosis was dramatically interrupted in MCT-induced PAH, which coincided to some extent with the features of the quasi-cancerous cells [45-49]. 


\section{Cellular Physiology Cell Physiol Biochem 2018;49:172-189

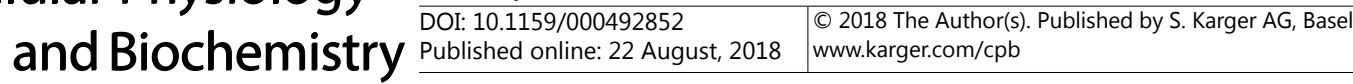

He et al.: Calcineurin/NFAT Signaling Modulates PASMC Proliferation, Migration and Apoptosis

Our data revealed that CaN/NFAT signaling was over-activated in MCT-PASMCs. The expression of CaNB $\beta / N F A T c 2-4$ was dramatically increased in MCT-reduced PAH rats. More specifically, protein expression of NFATc2-4 was significantly increased in the nucleus. These results were further demonstrated by the $27.70 \%, 28.48 \%$ and $55.02 \%$ increase in the nuclear to cytoplasmic ratio of NFATc2-4, respectively, as assessed by immunofluorescence, thus demonstrating enhanced nuclear localization. To further refine the effect of CaN/ NFAT on PASMCs, CsA, a nonselective pharmacological inhibitor of CaN, was subsequently applied to generally suppress the CaN/NFAT pathway. As shown in Fig. 5, CsA arrested cell proliferation and migration in both CON- and MCT-PASMCs in a dose-dependent manner. CsA appeared to exert a stronger inhibitory effect on the proliferation and migration of MCTPASMCs than on those of CON-PASMCs. The $\mathrm{IC}_{50}$ of proliferation inhibition was $23.29 \pm 1.09$ $\mu \mathrm{M}$ in MCT-PASMCs, which was $32.94 \%$ lower than that in CON-PASMCs $(34.73 \pm 2.23 \mu \mathrm{M})$. Similarly, the inhibition of MCT-PASMC migration was 1.59-, 1.29- and 1.08- times higher than that of CON-PASMC migration in the presence of 15, 30, and $60 \mu \mathrm{M}$ CsA, respectively. These data demonstrated effective attenuation of excessive PASMC proliferation and migration by CsA in MCT-induced PAH and thus support the involvement of CaN/NFAT in PAH.

The NFAT family is composed of five well-characterized members, NFATc1 (also known as NFAT2 or NFATx), NFATc2 (NFAT1 or NFATp), NFATc3 (NFAT4 or NFATx), NFATc4 (NFAT3) and NFAT5 (tonicity enhancer binding protein, TonEBP), as previously reviewed $[23,50]$. As the substrates of CaN, each member of NFAT family usually has different roles, especially in different physiological and pathophysiological states [20, 25, 29-31, 51]. We further examined the NFAT family members, with the expectation of identifying molecular targets that are responsible for the MCT-mediated induction of PAH. As presented in the paper, NFATc2-4 overexpression and nuclear translocation were observed in MCT-induced PAH. Moreover, siRNA targeting NFATc2-4 successfully reversed the excessive proliferation and migration as well as the apoptosis resistance in MCT-PASMCs. Notably, NFATc3 was the most effective member of the NFAT family on MCT-PASMCs. Silencing NFATc3 decreased PASMC proliferation by $36.43 \%$ according to the MTS assay and by $89.07 \%$ according to the PCNA expression analysis by immunofluorescence. Meanwhile, PASMC migration was reduced by $55.07 \%$ in MCT-induced PAH compared to controls. Most apparently, NFATc3 knockdown led to a two-fold increase in MCT-PASMC apoptosis compared to PASMC apoptosis in the mock group. Generally, our results showed that NFATc3 specifically acted on MCT-PASMCs, while silencing of NFATc2 and NFATc4 affected not only MCT-PASMCs but also CON-PASMCs. In addition, NFATc3 has been reported to regulate PASMC proliferation, migration and apoptosis in chronic hypoxia-induced PAH $[35,52]$. The above information suggests that NFATc3 might be an ideal therapeutic target for PAH due to its effectiveness and selectivity in PAH.

Interestingly, although, silencing NFATc2-4 enhanced PASMC apoptosis, inhibition of upstream CaN by CsA did not yield similar effects on apoptosis. CsA even blunted cell apoptosis at a dose of $60 \mu \mathrm{M}$. Due to its inhibition of CaN activity, CsA can subsequently obstruct the activation of the NFAT family, including not only NFATc2-4 (assessed in this study) but also NFATc1 and NFAT5. As shown in Fig. 2, NFATc1 and especially NFAT5 were highly expressed in PASMCs, but the roles of these proteins remain unclear. In addition, some researchers are of the opinion that CsA affects cell apoptosis in an NFAT-independent manner or by decreasing STAT3/NF- $\kappa$ B activation $[53,54]$. It is well known that NFAT is the most important substrate of CaN. However, other molecules, such as $\mathrm{Bad}, \mathrm{Na}^{+} / \mathrm{H}^{+}$exchanger, TWIK-related spinal cord potassium channel, and myocyte enhancer factor- 2 transcription factor, are all downstream of CaN [55-58]. Unfortunately, these signaling molecules have scarcely been studied in $\mathrm{PAH}$, and thus, little is known about their roles. Considering the possible involvement of these uncertain factors, the effect of CaN silencing is likely different from that of specifically silencing NFATc2-4 with siRNA.

It is noteworthy that the present study was carried out using in vitro cultured PASMCs originating from MCT-induced PAH rats. An obvious limitation of in vitro studies is the lack of the complexity of animal models or the human body. Moreover, the differences between MCT models and human PAH should also be taken into consideration. MCT not only induces 


\section{Cellular Physiology Cell Physiol Biochem 2018;49:172-189 \\ \begin{tabular}{l|l|l} 
DOI: 10.1159/000492852 & $\begin{array}{l}\text { O 2018 The Author(s). Published by S. Karger AG, Basel } \\
\text { www.karger.com/cpb }\end{array}$
\end{tabular}}

He et al.: Calcineurin/NFAT Signaling Modulates PASMC Proliferation, Migration and Apoptosis

PAH in rats but also affects both the right and left ventricles and other organs such as the liver and kidney, which notably increases mortality [59,60]. Nevertheless, MCT-induced PAH appears to be curable by several agents in rats, which is different from PAH in humans [39]. Moreover, PAH-induced vascular remodeling results from interactions between the different cell types of the pulmonary artery wall, which include not only smooth muscle cells but also endothelial cells and fibroblasts. Therefore, it is best to regard our results as a crude test of our hypothesis, which should be further verified in several other PAH models, including human PASMCs and NFAT KO mice. Although our data suggested the involvement of CaN/ NFAT signaling in the proliferation, migration and apoptosis of PASMCs in PAH, the effect of the entire signaling pathway cannot be fully elucidated without understanding NFAT target genes and NFAT-regulated- proteins. Thus, subsequent in vivo research and identification of the downstream effectors of NFAT are indubitably required to define the role of CaN/NFAT signaling in vascular remodeling in PAH.

In summary, this paper presents evidence indicating that CaN/NFAT signaling is involved in the aberrant proliferation, migration and apoptosis of PASMCs in MCT-induced PAH. NFATc2 and NFATc4 are essential for PASMC growth, migration and apoptosis. More importantly, NFATc3 plays a uniquely significant role in the disruption of PASMC proliferation in PAH. Thus, CaN/NFAT signaling may contribute to the progression of MCT-induced PAH and merits further study.

\section{Acknowledgements}

This work was supported by grants from the National Natural Science Foundation of China (NSFC31571179, NSFC31371165 and NSFC81400236), Natural Science Foundation of Fujian Province (2017J05134), Fujian Province "Hundred Experts Award" and Nursery Fund of Fujian Medical University (2014MP015).

\section{Disclosure Statement}

The authors declare that there is no conflict of interests.

\section{References}

1 Simonneau G, Gatzoulis MA, Adatia I, Celermajer D, Denton C, Ghofrani A, Gomez Sanchez MA, Krishna Kumar R, Landzberg M, Machado RF, Olschewski H, Robbins IM, Souza R: Updated clinical classification of pulmonary hypertension. J Am Coll Cardiol 2013;62:D34-41.

-2 Petersen C, Helvind M, Jensen T, Andersen HO: Potts shunt in a child with end-stage pulmonary hypertension after late repair of ventricular septal defect. World J Pediatr Congenit Heart Surg 2013;4:286289.

-3 Rosenkranz S, Gibbs JS, Wachter R, De Marco T, Vonk-Noordegraaf A, Vachiery JL: Left ventricular heart failure and pulmonary hypertension. Eur Heart J 2016;37:942-954.

-4 Shimoda LA, Laurie SS: Vascular remodeling in pulmonary hypertension. J Mol Med 2013;91:297-309.

-5 Thompson AA, Lawrie A: Targeting Vascular Remodeling to Treat Pulmonary Arterial Hypertension. Trends Mol Med 2017;23:31-45.

-6 Tuder RM, Archer SL, Dorfmuller P, Erzurum SC, Guignabert C, Michelakis E, Rabinovitch M, Schermuly R, Stenmark KR, Morrell NW: Relevant issues in the pathology and pathobiology of pulmonary hypertension. J Am Coll Cardiol 2013;62:D4-12.

7 Zhang S, Fantozzi I, Tigno DD, Yi ES, Platoshyn O, Thistlethwaite PA, Kriett JM, Yung G, Rubin LJ, Yuan JX: Bone morphogenetic proteins induce apoptosis in human pulmonary vascular smooth muscle cells. Am J Physiol Lung Cell Mol Physiol 2003;285:L740-754. 


\section{Cellular Physiology Cell Physiol Biochem 2018;49:172-189 \begin{tabular}{ll|l} 
aOI: 10.1159/000492852 & $\begin{array}{l}\text { O 2018 The Author(s). Published by S. Karger AG, Basel } \\
\text { www.karger.com/cpb }\end{array}$ \\
\hline
\end{tabular}}

He et al.: Calcineurin/NFAT Signaling Modulates PASMC Proliferation, Migration and Apoptosis

8 McMurtry MS, Archer SL, Altieri DC, Bonnet S, Haromy A, Harry G, Puttagunta L, Michelakis ED: Gene therapy targeting surviving selectively induces pulmonary vascular apoptosis and reverses pulmonary arterial hypertension. J Clin Invest 2005;115:1479-1491.

-9 Liu XR, Zhang MF, Yang N, Liu Q, Wang RX, Cao YN, Yang XR, Sham JS, Lin MJ: Enhanced store-operated $\mathrm{Ca}^{2+}$ entry and TRPC channel expression in pulmonary arteries of monocrotaline-induced pulmonary hypertensive rats. Am J Physiol Cell Physiol 2012;302:C77-87.

10 Xu Y, Bei Y, Shen S, Zhang J, Lu Y, Xiao J, Li X: MicroRNA-222 Promotes the Proliferation of Pulmonary Arterial Smooth Muscle Cells by Targeting P27 and TIMP3. Cell Physiol Biochem 2017;43:282-292.

$\$ 11$ Benza RL, Miller DP, Barst RJ, Badesch DB, Frost AE, McGoon MD: An evaluation of long-term survival from time of diagnosis in pulmonary arterial hypertension from the REVEAL Registry. Chest 2012;142:448-456.

12 Rhodes CJ, Davidson A, Gibbs JS, Wharton J, Wilkins MR: Therapeutic targets in pulmonary arterial hypertension. Pharmacol Ther 2009;121:69-88.

13 Lipskaia L, del Monte F, Capiod T, Yacoubi S, Hadri L, Hours M, Hajjar RJ, Lompre AM: Sarco/endoplasmic reticulum $\mathrm{Ca}^{2+}$-ATPase gene transfer reduces vascular smooth muscle cell proliferation and neointima formation in the rat. Circ Res 2005;97:488-495.

14 Fathi AT, Arowojolu O, Swinnen I, Sato T, Rajkhowa T, Small D, Marmsater F, Robinson JE, Gross SD, Martinson M, Allen S, Kallan NC, Levis M: A potential therapeutic target for FLT3-ITD AML: PIM1 kinase. Leuk Res 2012;36:224-231.

15 Song S, Yamamura A, Yamamura H, Ayon RJ, Smith KA, Tang H, Makino A, Yuan JX: Flow shear stress enhances intracellular $\mathrm{Ca}^{2+}$ signaling in pulmonary artery smooth muscle cells from patients with pulmonary arterial hypertension. Am J Physiol Cell Physiol 2014;307:C373-383.

-16 Weir EK, Cabrera JA, Mahapatra S, Peterson DA, Hong Z: The role of ion channels in hypoxic pulmonary vasoconstriction. Adv Exp Med Biol 2010;661:3-14.

17 Zhang S, Dong H, Rubin LJ, Yuan JX: Upregulation of $\mathrm{Na}^{+} / \mathrm{Ca}^{2+}$ exchanger contributes to the enhanced $\mathrm{Ca}^{2+}$ entry in pulmonary artery smooth muscle cells from patients with idiopathic pulmonary arterial hypertension. Am J Physiol Cell Physiol 2007;292:C2297-2305.

18 Earley S BJ: Transient receptor potential channels in the vasculature. Physiol Rev 2015;95:645-690.

-19 Potier M GJ, Motiani RK, Abdullaev IF, Bisaillon JM, Singer HA, Trebak M: Evidence for stim1- and orai1dependent store-operated calcium influx through $I_{\text {CRAC }}$ in vascular smooth muscle cells: Role in proliferation and migration. FASEB J 2009;232:2425-2437.

20 Quang CT, Leboucher S, Passaro D, Fuhrmann L, Nourieh M, Vincent-Salomon A, Ghysdael J: The calcineurin/NFAT pathway is activated in diagnostic breast cancer cases and is essential to survival and metastasis of mammary cancer cells. Cell Death Dis 2015;6:e1658.

-21 Soudani N, Ghantous CM, Farhat Z, Shebaby WN, Zibara K, Zeidan A: Calcineurin/NFAT ActivationDependence of Leptin Synthesis and Vascular Growth in Response to Mechanical Stretch. Front Physiol 2016;7:433.

-22 Benavides Damm T, Egli M: Calcium's role in mechanotransduction during muscle development. Cell Physiol Biochem 2014;33:249-272.

-23 Zhao L, Sullivan MN, Chase M, Gonzales AL, Earley S: Calcineurin/nuclear factor of activated T cells-coupled vanilliod transient receptor potential channel $4 \mathrm{Ca}^{2+}$ sparklets stimulate airway smooth muscle cell proliferation. Am J Respir Cell Mol Biol 2014;50:1064-1075.

-24 Fric J, Lim CX, Koh EG, Hofmann B, Chen J, Tay HS, Mohammad Isa SA, Mortellaro A, Ruedl C, RicciardiCastagnoli P: Calcineurin/NFAT signalling inhibits myeloid haematopoiesis. EMBO Mol Med 2012;4:269282.

25 Serrano-Perez MC, Fernandez M, Neria F, Berjon-Otero M, Doncel-Perez E, Cano E, Tranque P: NFAT transcription factors regulate survival, proliferation, migration, and differentiation of neural precursor cells. Glia 2015;63:987-1004.

-26 Qin JJ, Nag S, Wang W, Zhou J, Zhang WD, Wang H, Zhang R: NFAT as cancer target: mission possible? Biochim Biophys Acta 2014;1846:297-311.

27 Chen R, Yan J, Liu P, Wang Z, Wang C, Zhong W, Xu L: The role of nuclear factor of activated T cells in pulmonary arterial hypertension. Cell Cycle 2017;16:508-514.

28 Nilsson LM, Nilsson-Ohman J, Zetterqvist AV, Gomez MF: Nuclear factor of activated T-cells transcription factors in the vasculature: the good guys or the bad guys? Curr Opin Lipidol 2008;19:483-490. 


\section{Cellular Physiology Cell Physiol Biochem 2018;49:172-189 \begin{tabular}{l|l|l} 
and Biochemistry Published online: 22 August, 2018 & $\begin{array}{l}\text { @ } 2018 \text { The Author(s). Published by S. Karger AG, Basel } \\
\text { www.karger.com/cpb }\end{array}$ \\
\hline
\end{tabular}}

He et al.: Calcineurin/NFAT Signaling Modulates PASMC Proliferation, Migration and Apoptosis

29 Barzegar-Fallah A, Alimoradi H, Razmi A, Dehpour AR, Asgari M, Shafiei M: Inhibition of calcineurin/ NFAT pathway plays an essential role in renoprotective effect of tropisetron in early stage of diabetic nephropathy. Eur J Pharmacol 2015;767:152-159.

-30 Mojsa B, Mora S, Bossowski JP, Lassot I, Desagher S: Control of neuronal apoptosis by reciprocal regulation of NFATc3 and Trim17. Cell Death Differ 2015;22:274-286.

31 Li M, He HP, Gong HQ Zhang J, Ma WJ, Zhou H, Cao DS, Wang N, Zhang TC: NFATc4 and myocardin synergistically up-regulate the expression of LTCC alpha1C in ET-1-induced cardiomyocyte hypertrophy. Life Sci 2016;155:11-20.

32 Perros F, Dorfmuller P, Montani D, Hammad H, Waelput W, Girerd B, Raymond N, Mercier O, Mussot S, Cohen-Kaminsky S, Humbert M, Lambrecht BN: Pulmonary lymphoid neogenesis in idiopathic pulmonary arterial hypertension. Am J Respir Crit Care Med 2012;185:311-321.

-33 Bonnet S, Rochefort G, Sutendra G, Archer SL, Haromy A, Webster L, Hashimoto K, Bonnet SN, Michelakis ED: The nuclear factor of activated T cells in pulmonary arterial hypertension can be therapeutically targeted. Proc Natl Acad Sci USA 2007;104:11418-11423.

-34 Wang C, Li JF, Zhao L, Liu J, Wan J, Wang YX, Wang J, Wang C: Inhibition of SOC/Ca ${ }^{2+} / \mathrm{NFAT}_{\text {pathway is }}$ involved in the anti-proliferative effect of sildenafil on pulmonary artery smooth muscle cells. Respir Res 2009;10:123.

35 de Frutos S, Spangler R, Alo D, Bosc LV: NFATc3 mediates chronic hypoxia-induced pulmonary arterial remodeling with alpha-actin up-regulation. J Biol Chem 2007;282:15081-15089.

36 Kang K, Peng X, Zhang X, Wang Y, Zhang L, Gao L, Weng T, Zhang H, Ramchandran R, Raj JU, Gou D, Liu L: MicroRNA-124 suppresses the transactivation of nuclear factor of activated $T$ cells by targeting multiple genes and inhibits the proliferation of pulmonary artery smooth muscle cells. J Biol Chem 2013;288:25414-25427.

-37 Hou X, Chen J, Luo Y, Liu F, Xu G, Gao Y: Silencing of STIM1 attenuates hypoxia-induced PASMCs proliferation via inhibition of the SOC/Ca ${ }^{2+} / \mathrm{NFAT}$ pathway. Respir Res 2013;14:2.

38 Wang RX, He RL, Jiao HX, Dai M, Mu YP, Hu Y, Wu ZJ, Sham JS, Lin MJ: Ginsenoside Rb1 attenuates agonistinduced contractile response via inhibition of store-operated calcium entry in pulmonary arteries of normal and pulmonary hypertensive rats. Cell Physiol Biochem 2015;35:1467-1481.

-39 Stenmark KR, Meyrick B, Galie N, Mooi WJ, McMurtry IF: Animal models of pulmonary arterial hypertension: the hope for etiological discovery and pharmacological cure. Am J Physiol Lung Cell Mol Physiol 2009;297:L1013-1032.

40 Sztuka K, Jasinska-Stroschein M: Animal models of pulmonary arterial hypertension: A systematic review and meta-analysis of data from 6126 animals. Pharmacol Res 2017;125:201-214.

41 Mu YP, Lin DC, Yan FR, Jiao HX, Gui LX, Lin MJ: Alterations in Caveolin-1 Expression and Receptor-Operated $\mathrm{Ca}^{2+}$ Entry in the Aortas of Rats with Pulmonary Hypertension. Cell Physiol Biochem 2016;39:438-452.

$>42$ Lin MJ, Leung GP, Zhang WM, Yang XR, Yip KP, Tse CM, Sham JS: Chronic hypoxia-induced upregulation of store-operated and receptor-operated $\mathrm{Ca}^{2+}$ channels in pulmonary arterial smooth muscle cells: a novel mechanism of hypoxic pulmonary hypertension. Circ Res 2004;95:496-505.

43 Sun Z, Nie X, Sun S, Dong S, Yuan C, Li Y, Xiao B, Jie D, Liu Y: Long Non-Coding RNA MEG3 Downregulation Triggers Human Pulmonary Artery Smooth Muscle Cell Proliferation and Migration via the p53 Signaling Pathway. Cell Physiol Biochem 2017;42:2569-2581.

44 Zhou Y, Wang Y, Wang X, Tian X, Zhang S, Yang F, Guo H, Fan R, Feng N, Jia M, Gu X, Wang Y, Li J, Pei J: The Protective Effects of Kappa-Opioid Receptor Stimulation in Hypoxic Pulmonary Hypertension Involve Inhibition of Autophagy Through the AMPK-MTOR Pathway. Cell Physiol Biochem 2017;44:1965-1979.

45 Boucherat 0, Vitry G, Trinh I, Paulin R, Provencher S, Bonnet S: The cancer theory of pulmonary arterial hypertension. Pulm Circ 2017;7:285-299.

46 Rai PR, Cool CD, King JA, Stevens T, Burns N, Winn RA, Kasper M, Voelkel NF: The cancer paradigm of severe pulmonary arterial hypertension. Am J Respir Crit Care Med 2008;178:558-564.

47 Dromparis P, Paulin R, Sutendra G, Qi AC, Bonnet S, Michelakis ED: Uncoupling protein 2 deficiency mimics the effects of hypoxia and endoplasmic reticulum stress on mitochondria and triggers pseudohypoxic pulmonary vascular remodeling and pulmonary hypertension. Circ Res 2013;113:126-136.

-48 Xiao Y, Peng H, Hong C, Chen Z, Deng X, Wang A, Yang F, Yang L, Chen C, Qin X: PDGF Promotes the Warburg Effect in Pulmonary Arterial Smooth Muscle Cells via Activation of the PI3K/AKT/mTOR/HIF-1alpha Signaling Pathway. Cell Physiol Biochem 2017;42:1603-1613. 


\section{Cellular Physiology Cell Physiol Biochem 2018;49:172-189

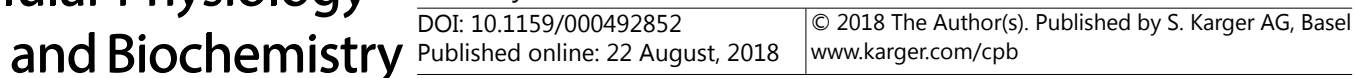

He et al.: Calcineurin/NFAT Signaling Modulates PASMC Proliferation, Migration and Apoptosis

49 Yu L, Tu Y, Jia X, Fang K, Liu L, Wan L, Xiang C, Wang Y, Sun X, Liu T, Yu D, Cao W, Song Y: Resveratrol Protects Against Pulmonary Arterial Hypertension in Rats via Activation of Silent Information Regulator 1 Cell Physiol Biochem 2017;42:55-67.

50 Muller MR, Rao A: NFAT, immunity and cancer: a transcription factor comes of age. Nat Rev Immunol 2010;10:645-656.

51 Zaslavsky A, Chou ST, Schadler K, Lieberman A, Pimkin M, Kim YJ, Baek KH, Aird WC, Weiss MJ, Ryeom S: The calcineurin-NFAT pathway negatively regulates megakaryopoiesis. Blood 2013;121:3205-3215.

52 Bierer R, Nitta CH, Friedman J, Codianni S, de Frutos S, Dominguez-Bautista JA, Howard TA, Resta TC, Bosc LV: NFATc3 is required for chronic hypoxia-induced pulmonary hypertension in adult and neonatal mice. Am J Physiol Lung Cell Mol Physiol 2011;301:L872-880.

53 Shou J, You L, Yao J, Xie J, Jing J, Jing Z, Jiang L, Sui X, Pan H, Han W: Cyclosporine A sensitizes human nonsmall cell lung cancer cells to gefitinib through inhibition of STAT3. Cancer Lett 2016;379:124-133.

54 Werneck MB, Hottz E, Bozza PT, Viola JP: Cyclosporin A inhibits colon cancer cell growth independently of the calcineurin pathway. Cell Cycle 2012;11:3997-4008.

55 Li G, Yan W, Dang Y, Li J, Liu C, Wang J: The role of calcineurin signaling in microcystin-LR triggered neuronal toxicity. Sci Rep 2015;5:11271.

56 Allman E, Waters K, Ackroyd S, Nehrke K: Analysis of $\mathrm{Ca}^{2+}$ signaling motifs that regulate proton signaling through the $\mathrm{Na}^{+} / \mathrm{H}^{+}$exchanger NHX-7 during a rhythmic behavior in Caenorhabditis elegans. J Biol Chem 2013;288:5886-5895.

-57 Li J, Vargas MA, Kapiloff MS, Dodge-Kafka KL: Regulation of MEF2 transcriptional activity by calcineurin/ mAKAP complexes. Exp Cell Res 2013;319:447-454.

58 Czirjak G, Enyedi P: The LQLP calcineurin docking site is a major determinant of the calcium-dependent activation of human TRESK background $\mathrm{K}^{+}$channel. J Biol Chem 2014;289:29506-29518.

59 Kolli MB, Manne N, Para R, Nalabotu SK, Nandyala G, Shokuhfar T, He K, Hamlekhan A, Ma JY, Wehner PS, Dornon L, Arvapalli R, Rice KM, Blough ER: Cerium oxide nanoparticles attenuate monocrotaline induced right ventricular hypertrophy following pulmonary arterial hypertension. Biomaterials 2014;35:99519962.

60 Yao J, Li CG, Gong LK, Feng CC, Li CZ, Gao M, Luan Y, Qi XM, Ren J: Hepatic cytochrome P450s play a major role in monocrotaline-induced renal toxicity in mice. Acta Pharmacol Sin 2014;35:292-300. 